PALEO

Revue d'archéologie préhistorique

$18 \mid 2006$

Varia

\title{
Petit gibier et fonction de sites au Paléolithique supérieur
}

Les ensembles fauniques de la grotte d'Anecrial (Porto de Mos, Estremadure, Portugal)

Small game and site function in upper Paleolithic: the faunal assemblages from the cave of Anecrial (Porto de Mos, Estrémadure, Portugal)

Jean-Philip Brugal

\section{OpenEdition \\ Journals}

Édition électronique

URL : http://journals.openedition.org/paleo/140

DOI : 10.4000/paleo.140

ISSN : 2101-0420

Éditeur

SAMRA

Édition imprimée

Date de publication : 1 décembre 2006

Pagination : 45-68

ISSN : 1145-3370

Référence électronique

Jean-Philip Brugal, « Petit gibier et fonction de sites au Paléolithique supérieur », PALEO [En ligne], 18| 2006, mis en ligne le 23 avril 2009, consulté le 07 juillet 2020. URL : http://journals.openedition.org/ paleo/140 ; DOI : https://doi.org/10.4000/paleo.140

\section{(c) (i) (2)}

PALEO est mis à disposition selon les termes de la licence Creative Commons Attribution - Pas d'Utilisation Commerciale - Pas de Modification 4.0 International. 


\title{
PETIT GIBIER ET FONCTION DE SITES AU PALÉOLITHIQUE SUPÉRIEUR : LES ENSEMBLES FAUNIQUES DE LA GROTTE D'ANECRIAL (Porto de Mos, Estremadure, Portugal)
}

\author{
Jean-Philip BRUGAL ${ }^{(1)}$
}

\begin{abstract}
Résumé : Le gisement en grotte d'Anecrial livre des ensembles fauniques dominés par les lagomorphes (genre Oryctolagus), représentés par peu d'individus. La dispersion des restes, les parties du squelette et les types de fracturation (cylindres) précisent l'origine de l'accumulation et les hypothèses fonctionnelles du site. Le gisement est interprété comme un bivouac utilisé au moins à trois reprises par un groupe humain réduit, orienté vers l'acquisition spécifique de ce petit gibier. La comparaison avec d'autres gisements met en évidence l'importance des Léporidés dans l'alimentation des hommes du Paléolithique supérieur moyen et final, en particulier au Portugal, et aborde les relations entre des facteurs environnementaux et des modèles socio-économiques humains.
\end{abstract}

Mot-clés : Paléolithique supérieur, Lagomorphes, Taphonomie, Fonction de sites, Économie Key-words : Upper Palaeolithic, Lagomorphe, Taphonomy, Site function, Economy

\author{
Abridged english version \\ SMALL GAME AND SITE FUNCTION IN UPPER PALEOLITHIC : \\ THE FAUNAL ASSEMBLAGES FROM THE CAVE OF ANECRIAL (Porto de Mos, Estrémadure, Portugal)
}

\begin{abstract}
Inferences about socio-economic human behaviors need to integrate all sources of data coming from cave and open-air archaeological sites, and from abundant to poor-dense levels/sites. They have also to interface lithic productions and subsistence strategies. These approaches are especially relevant during the Upper Palaeolithic where a diet enlargement, focused on small game acquisition, is observed, combined with technological innovations. The appearance and/or diffusion of such processes have to be précised at the scale of Mediterranean realm. In this context, the cave site of Anecrial in central Portuguese Estremadura brings new insights about the human variability of leporid acquisition. Lapa do Anecrial is a small cave (fig.1) which has provided a stratigraphic sequence spanning the transition from the Gravettian to the Solutrean, with three short-term occupations recognized: one in layer 1 with very few solutrean artifacts dated to $20520 \pm 100 \mathrm{BP}$, and two in layer 2 . The layer 2 is a surface $\left(9.5 \mathrm{~m}^{2}\right)$ dated between $\sim 23600 \mathrm{BP}$ and $21600 \mathrm{BP}$ which seems to have been stable with scarce sediment input, with two Gravettian occupations detected. One concerns remains of an older occupation with rolled artifacts and few ibex bones while the other shows better post-depositional integrity, with several clusters of lithic artifacts attributed to Proto-Solutrean/Terminal Gravettian and a fauna massively dominated by rabbit; remains are organized around a hearth en cuvette. The lithic refitting studies (almost $50 \%$ - and $92 \%$ in weight - refitted) and spatial distribution (Almeida et al. ss-pr.) confirm the excellent preservation of this occupation, making possible an almost complete view of the reduction sequences and strategies applied to the lithics. The sedimentology,
\end{abstract}

(1) Maison Méditerranéenne des Sciences de l'Homme, UMR 6636 “Economies, Sociétés, Environnements Préhistoriques ", BP 647 F-13094 Aix-en-Provence - brugal@mmsh.univ-aix.fr 
anthracology and carbon dating precise the paleoenvironment dominated by an open landscape with Leguminosae and some Pine and heath under a rather cool climate. The bone assemblage is mainly composed of rabbit (genus Oryctolagus, fig. 2) from the layer 1 ( $n=214$ which 15 ibex bones) and the layer 2 ( $n=1516$ which 75 ibex bones) (tabl.1), closely found around and in the fireplaces. The ibex remains (tabl.2) are weathered, chalky and white, with very few identifiable elements (distal humerus, pelvis fragment metatarsal shaft, no teeth) and chips dominant (average length of $37 \mathrm{~mm}$ ) showing dry breakage. Cutmarks are present $(n=9)$ as well as dissolution marks (fig.3) and one small carnivore marks. They are interpreted as the result of reworked material from an ancient occupation (Gravettian, from the dating caprid sample), mixed with most of the faunal and lithic finds exposed in these layers. The rabbit material is the most abundant with 1600 bones and teeth, distributed in 4 individuals in layer 1 and 15 in layer 2, mostly adults (fig.4). They correspond to poor-dense site in comparison with some other upper Paleolithic sites which yield thousands remains and hundred individuals of leporid. At Anecrial, all skeletal parts are present, with a good balance between right and left elements that evidence the inflow of complete carcasses (tabl.3 et 4). The preservation state is relatively good, with little evidence of dissolution, root marks, carnivore puncture and more anecdotic, gastropod activities (fig.5); black burnt materials represent $17 \%$ in layer 2 . Only one cut mark is visible from layer 2 (metapodial) and four pelvis fragment show traces possibly reported to human teeth. Some discrepancy occurs in skeletal elements with under representation of axial and distal legs (carpal/tarsal, metapodials, phalanx; tabl.5) and many upper long bone shafts (tibia, femur, humerus), typical of human action for marrow extraction (fig.6); long bone cylinders are dominant (with long fragment vs short ones; tabl.6 et fig.7) and epiphysis ends are systematically missing which could indicate relatively low postdepositional perturbation and heavy human consumption. Moreover, these percentage disparities between some of the elements suggest differential human used. The absence of distal member parts can be explained by hide curation, the extremities of the legs being transported together with the rabbit hides. The low presence of axial parts, however, is harder to explain. A possible hypothesis, that deserves further research as ethnographic cases, could be a different consumption technique, like smashing the bones and inner parts into a greasy rich mass. Finally, the spatial distribution with dense cluster around hearth demonstrates synchronicity of prehistoric activities for each layer.

The Leporid bone assemblage from upper Paleolithic levels of Anecrial can deserve further taphonomic and palethnography investigations. Small species accumulations in archeological context may have several origins, from natural mortality (in situ attrition) to predations s.I.: for instance small to medium sized carnivores as red fox or iberic lynx (this last one with around $88 \%$ of rabbit remains in faeces from present observations), which are frequent in Iberian Paleolithic faunal record. Many specialized literature concern the formation processes of small mammal bone accumulation, with neotaphonomical informations as well as studies from archaeological context and some ethnographic examples. They allow developing different parameters: skeletal representation and breakage patterns, age structure, marks s.l., spatial distribution. The presence of shaft fragments (cylinders) of long bones, especially femur, tibia, humerus, are a good signature of anthropic activities, for marrow extraction. It seems that a most common hunting strategy of rabbit need the use of trap in the close vicinity of burrows, as suggested in the Magdalenian site of Picareiro, not far from Anecrial, where huge amounts of rabbit bone accumulations are present. At this point, a comparative examination of number of leporid in the fossil to recent accumulations show two main categories: one with relatively few remains (less than 1500-2000) either constituted by natural or anthropic actions (it is the case for Anecrial) and the second with quite more 2 000 remains, all make up by humans. The latter concerns sites from the end of European Upper Paleolithic (late Magdalenian and Epipaleolithic-Mesolithic), where some very rich localities, dominated by hare (genus Lepus) or rabbit (genus Oryctolagus, abundant in Iberia) are recognized (fig.8). The site function and the complete list of recorded taxa are important points to be considered in order to precise the role of small game into the subsistence economy of past human groups. Anecrial is a remarkable example with clear human involvement, no other game but few rabbit individuals. They demonstrate very short term, and unique, occupations in the course of human mobility with logistical purposes (transient camp?). The group could be of family-sized (mini. 3 to maxi. 5-6 persons) moving on a seasonal basis. The limited occupied space near the cave wall, the presence of three welldefined knapping-posts around the earth (Almeida et al, ss-pr.) and the small number of materials (rabbits and lithics) can support the site-function interpretation (fig.9 : level 2). The nature of such occupations is connected with the climatic environment, and the dates from the level 2 is contemporary of cold event known in marine record as Heinrich event $\mathrm{H} 2$; they are short duration cold period, between 500 to 2000 years, enough to unbalance the zoocoenose. During this time period, few other cave sites are present in Estremadura (Buraca Escura, Caldeirao, Salemas) and all of them indicate short human occupations in small cavities; an exception is the rock-shelter of Lagar Velho with more diversified faunas, dominated by rabbits, although represented by few specimens/individuals and environmental data (charcoal) suggesting also open landscape. The species associations in Portugal between 22-18 ka are composed of red deer and horse, followed by wild goat and aurochs; among carnivores, lynx and red fox, then wolf are present and large predators (lion, cave hyena) start to disappear. It is this same period, as a colder phase, where human groups develop small game exploitation, which can probably be related with demographic expansion of leporids and decrease (or more scattered) of ungulates.

The cave site of Anecrial yields two main archaeological levels, attributed to terminal Gravettian and Solutrean, characterized by relatively few lithic and faunal remains scattered on a limited surface around hearth structure. Faunal remains are mainly rabbit bones and we can notice the good integrity of assemblages. Such 'poor' sites are much informative about human activities integrated in socio-economic patterns and climatic environment. The site function can be précised with palethnographical interpretations through the analysis of skeletal representation, breakage patterns, burnt bones and spatial distribution. The example of Anecrial brings new insights about human subsistence variability toward specific resource, especially on small prey. They rise up the question of logistical relation in term of duration and seasonal human occupations (mobility and size of the groups) in connection with environmental (local?) factors. Then it becomes essential to well functionally determine each archaeological level before to propose a large-scale behaviorally overview about small game subsistence by Palaeolithic people. 
Les gisements préhistoriques sont de nature variée, se déclinant schématiquement en sites de plein-air et en cavités, livrant des séries lithiques et biologiques (faune, flore) plus ou moins denses et diversifiées, organisées ou non dans un espace domestique. Pour chacun de ses points géotopographique ; diversité, densité et distribution des séries ; présence de structures s.l. -, il est possible de définir plusieurs paramètres dont la conjugaison globale permet d'inférer des hypothèses fonctionnelles sur les lieux d'activités humaines en relation avec les environnements bioclimatiques. Les systèmes socio-économiques de groupes chasseurs-collecteurs peuvent ensuite être déduits d'un ensemble de sites de fonction(s) reconnue(s) pour une région et une culture. Afin de reconstruire ces systèmes, il est alors nécessaire de reconnaître toutes les catégories de gisements, depuis les plus denses - généralement connus et fouillés depuis longtemps (souvent en cavités) - jusqu'aux sites parfois considérés comme mineurs mais riches d'informations complémentaires. Ces gisements qualifiés de "pauvres" ${ }^{2}$ peuvent en effet apporter des renseignements plus détaillés car ils correspondent souvent à des durées et des intensités d'occupations limitées permettant d'approcher la synchronicité des activités humaines ; c'est le cas pour le gisement portugais d'Anecrial montrant deux occupations différentes du Paléolithique supérieur (Solutréen et Gravettien Teminal).

Les recherches sur les comportements de subsistance complètent largement celles menées en particulier sur les productions lithiques. Ces deux registres restent les témoins majeurs des activités humaines. La complémentarité de telles études a d'ores et déjà apporté de très nombreuses informations sur les modes de vie des populations du Paléolithique supérieur en Europe de l'Ouest. Un des points particuliers de la fin de cette période, pour les régions du sud de l'Europe tout au moins, concerne le développement de l'exploitation des ressources alimentaires, passant par un élargissement des gibiers de petite taille (par exemple : Straus 1991 ; Grayson et Delpech 1998 ; Stiner et al. 1999 ; Stiner 2004 ; Hockett et Haws 2002 ; Cochard et Brugal 2004). S'ajoutant à l'acquisition traditionnelle des ongulés, les préhistoriques vont dorénavant tirer parti des domaines aquatiques (poissons, mollusques), aérien (oiseaux) et terrestres (lagomorphes, rongeurs, reptiles, etc.). Ces changements sont souvent mis en relation avec des innovations techniques allant de pair avec une complexité des organisations sociales (sédentarité, démographie, ethnicité, etc.). II reste toutefois à mieux définir l'apparition et les rythmes dans la gestion de ces nouvelles ressources selon les provinces biogéographiques et culturelles à l'échelle de l'Europe méridionale ou circum-méditerranéenne.

Dans ce contexte dynamique de recherches, la péninsule ibérique constitue une région intéressante, à la fois par ses peuplements animaux et humains (par exemple, Brugal et Yravedra Sainz de los Terreros 2005-2006), mais aussi dans ses adaptations culturelles en rapport avec des milieux bioclimatiques spécifiques. II est en effet reconnu que les impacts des périodes glaciaires sont relativement atténués dans cette partie de l'Europe, bien que des gradients climatiques puissent se manifester et compartimenter les écosystèmes, d'autant plus que cette région présente des reliefs importants. Parmi les petits gibiers, le lapin de garenne (genre Oryctolagus) trouve en péninsule ibérique un refuge naturel au cours du Pléistocène (e.g., Calou 1995, Hockett et Bicho 2000) et son abondance a pu entraîner une exploitation différenciée de la part des chasseurs paléolithiques. Le gisement d'Anecrial en Estrémadure portugaise fournit un nouvel exemple d'acquisition de lagomorphes (Leporidae) durant la fin du Paléolithique supérieur, documentant la variabilité des adaptations humaines dans le cadre de gestion du territoire (mobilité, taille des groupes, etc.). Un premier jeu d'hypothèses fonctionnelles a été proposé pour la couche 2 par J. Zilhao (1997), sur la base des caractéristiques préliminaires et de la distribution spatiale des outillages, des matières premières lithiques et des restes fauniques. Un petit groupe d'individus aurait séjourné durant une très faible durée dans la cavité, avec divers postes de travail (taille) organisés autour d'un foyer ; une exploitation locale de lapins est enregistrée durant ce séjour avec la possibilité d'apport extérieur de portions de bouquetins. L'apport des études taphonomiques et archéozoologiques permet alors de tester ces propositions et d'affiner les premières interprétations.

\section{Le gisement de Lapa do Anecrial}

Le site d'Anecrial désigne une grotte de relative petite dimension (en portugais : 'Lapa' ou 'Buraca') avec une salle unique (e.100 $\mathrm{m}^{2}$, hauteur maximum de $6 \mathrm{~m}$ ) et une entrée relativement étroite regardant vers le Nord (fig.1). Elle se positionne sur le bord d'un poljé (Alvados), dans l'Estrémadure portugaise (altitude $340 \mathrm{~m}$ ), qui représente une voie de passage naturelle entre le bassin du Taje et l'Atlantique. La plus grande partie de la surface de la cavité est recouverte d'une croûte stalagmitique et seule la partie ouest de la salle, le long de la paroi, contient un remplissage sédimentaire : il a fait l'objet de fouilles (19921993 et 1995) sur une surface de 9,5 $\mathrm{m}^{2}$ (Zilhao 1997 ; Zilhao et Almeida 1996 ; Almeida 2000 ; Almeida et al. sous-presse). La séquence stratigraphique, épaisse d'environ 1,5 m, montre cinq couches dont deux (couches 1 et 2) livrent des artefacts lithiques.

La couche 1 (épaisseur d'environ $25 \mathrm{~cm}$ ) est constituée par un éboulis sec d'éléments anguleux $(5-10 \mathrm{~cm})$ dans une matrice sableuse. Elle contient à son sommet une série archéologique très peu dense (deux pièces lithiques dont une préforme de pointe à cran; quatre coquilles marines de Littorina obtusata et 214 restes de faune) autour d'un foyer avec quelques charbons qui fournissent une date AMS de

(2) Nous attribuons ici une valeur essentiellement quantitative à ces termes de pauvre (mineur/petit) vs riche (majeur/grand) (par ex. Isaac et al. 1981) ; c'est-à-dire livrant des ensembles archéologiques peu abondants et peu diversifiés en nombre de vestiges versus des ensembles très abondants et diversifiés. 


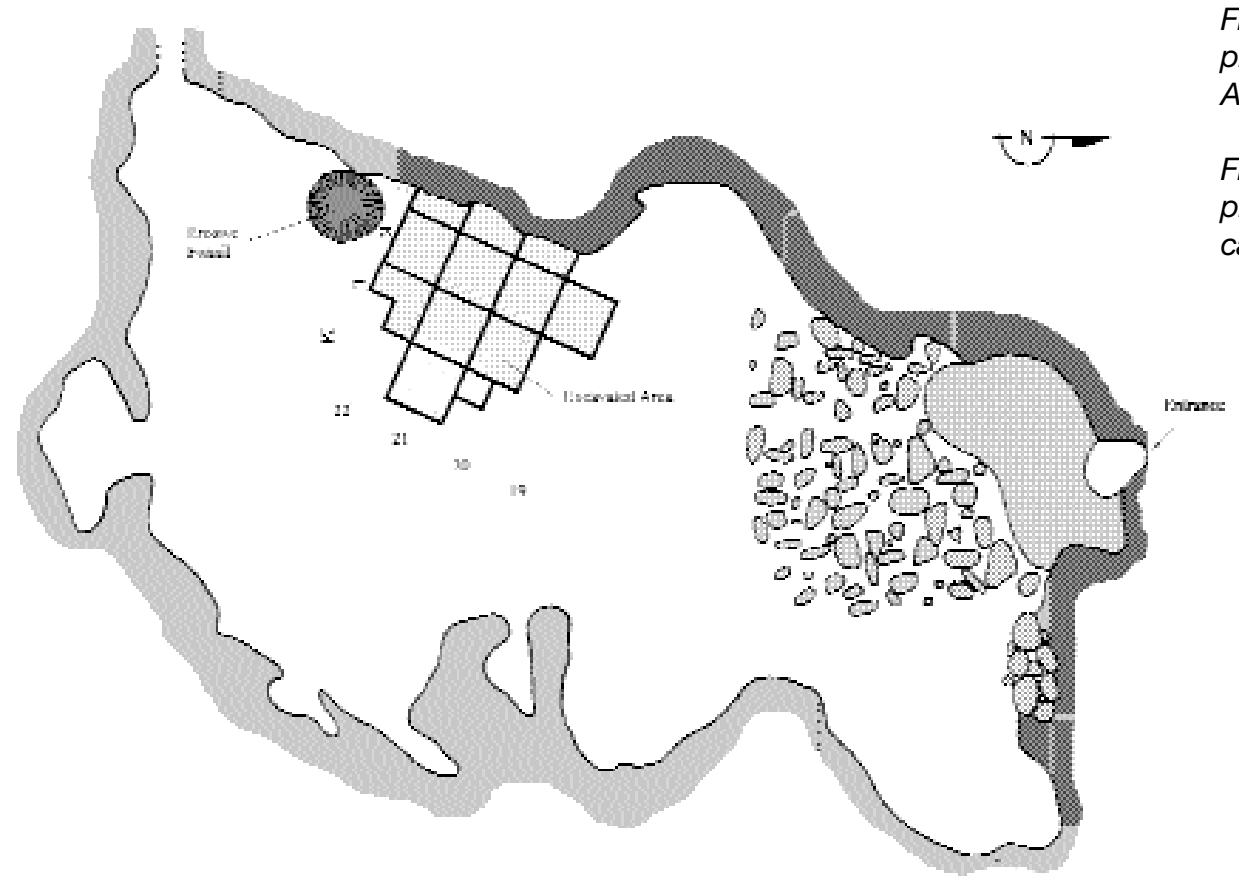

Figure 1 - Lapa do Anecrial : plan et profil de la grotte (d'après Almeida et al. ss-pr.).

Figure 1 - Lapa do Anecrial: plan and profile views of the cave (after Almeida et al. ss-pr.).

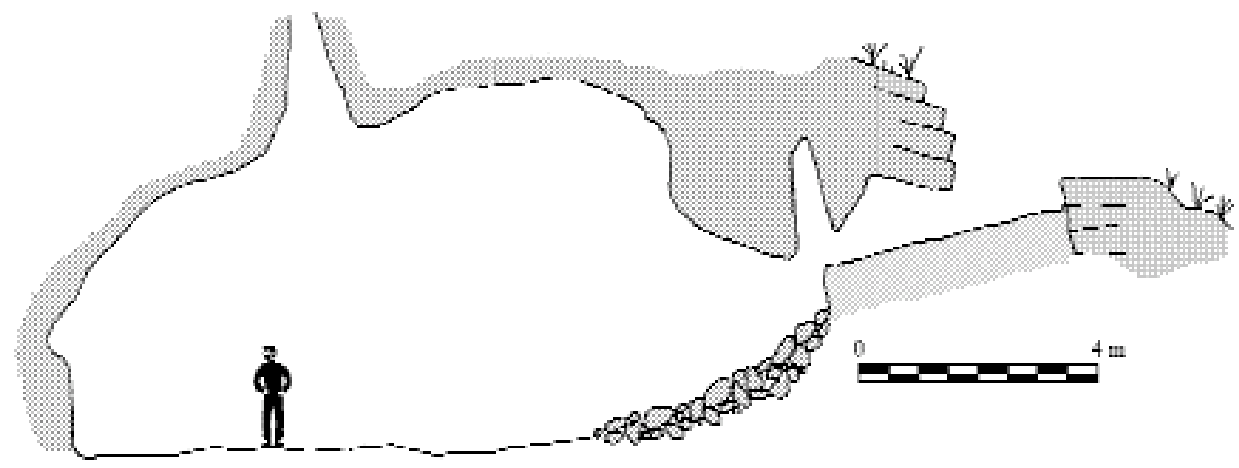

$20520 \pm 100$ ans BP (GrA-12019). Cette occupation est rapportée au Solutréen, contemporaine du dernier maximum glaciaire. On peut ajouter que le foyer, à plat, a un diamètre moyen de $70 \mathrm{~cm}$, contenant des charbons de pins (détermination I.Figueiral) et de nombreux restes de lapins.

La couche 2 (épaisseur d'environ $10 \mathrm{~cm}$ ) est composée d'un sable calcaire fin correspondant à un ralentissement dans la sédimentation. Un matériel plus abondant se répartit autour d'un foyer en cuvette (diamètre de $80 \mathrm{~cm}$ ) et l'ensemble présente une grande intégrité spatiale avec en particulier une distribution du lithique en groupes bien séparés de matières premières et de taille (présence de nucléus, éclats, racloirs, lamelles). Prés de 572 pièces lithiques (silex, quartz, quartzite) ont permis d'opérer de nombreux remontages (environ $51 \%$ et $92 \%$ en poids) (Almeida et al. sous-presse) qui démontrent une faible action post-dépositionnelle. Les études technologiques précisent les séquences de réduction et indiquent des activités de taille in situ notamment pour l'obtention d'armatures de petites dimensions (Zilhao 1997 ; Almeida 2000). L'assemblage faunique comprend de nombreux restes de lapins et quelques fragments de bouquetins distribués autour et dans le foyer. L'analyse anthracologique (I.Figueiral, inédit) montre la dominance de Légumineuses associées à du pin (Pinus sylvestris) et de la bruyère (Erica sp.). Les datations radiocarbones sur charbons fournissent un âge autour de 21600 ans BP [21560 \pm 680 ICEN-964 et AMS $21560 \pm$ 220 OxA-5526] alors qu'une date sur un os de bouquetin donne un âge AMS de $23410 \pm 170$ BP $(\mathrm{OxA}-11235)^{3}$. L'étude taphonomique permet en effet de rendre compte d'une altération différente entre les deux espèces de mammifères, ce qui supposait un mélange confirmé par ces datations. D'autre part, un petit stock de matériel lithique, correspondant à deux catégories de matières premières siliceuses, avait également un aspect plus roulé. Il est ainsi possible de reconnaître deux occupations préhistoriques dans ce niveau : le premier, le plus vieux et le plus dégradé,

(3) Cette date rejoint une autre datation 14C conventionnel à partir d'un charbon situé à la limite des couches 1 et 2 , montrant une forte marge d'erre ur : 23450 + 1470/- 1240 (ICEN-963). 


\begin{tabular}{|c|c|c|c|c|c|}
\hline couches & 0 & 1 & 2 & 3 & Total \\
\hline Gasléropodes & $60^{\circ}$ & 14 & 14 & 10 & 98 \\
\hline Pisces & 0 & 0 & 3 & 0 & 3 \\
\hline Amphibiens & 0 & 0 & 1 & 0 & 1 \\
\hline Aves & 0 & 1 & 6 & 0 & 7 \\
\hline Lagomorphes & 4 & 184 & 1417 & $6^{-x}$ & 1611 \\
\hline Caprinès & 0 & 15 & 75 & 0 & 90 \\
\hline NRD(=NISP) & 64 & 214 & 1516 & 16 & 1810 \\
\hline n carré & 4 & 7 & 10 & 3 & \\
\hline n carré Lago. & 3 & 7 & 10 & 3 & \\
\hline n carré Capr. & - & 4 & 8 & 3 & \\
\hline
\end{tabular}

Tableau 1 - Distribution stratigraphique et topographique (nombre de carrés) des espèces d'Anecrial (nombre de restes).

$n$ carré $=$ nombre de carré concerné et détail suivant les espèces (lagomorphes et caprinés).

Table 1 - Stratigraphic and topographic number of square) distribution of taxa from Anecrial (number of specimens).

rapporté au Gravettien et un deuxième, bien conservé, attribué au Proto-Solutréen/Gravettien terminal. L'analyse spatiale (verticale) confirme aussi cette interprétation avec les restes de bouquetin se plaçant à la base de la couche 2 , alors que la grande majorité des autres éléments sont en position supérieure (Almeida et al. sous-presse).

Une couche sus-jacente (c.0) paraît relativement hétérogè ne, composée de colluvions perturbées. Les couches sous-jacentes aux c. 1 et 2 sont archéologiquement stériles et le niveau 3n, avec un fragment de charbon, a pu être daté par AMS de $24410 \pm 110$ BP (GrA-12016). Ce sont des dépôts d'éboulis calcaire grossier (c.3 et 4), recouvrant un remplissage d'argile rouge stérile (c.5).

La grotte d'Anecrial documente des occupations humaines de courte durée de la fin du Gravettien et du début du Solutréen. Les données sur la nature sédimentaire et les résultats anthracologiques (Figueiral 1995 ; Figueiral et Te rral 2002 ; Queiroz et al. 2002 ; Zilhao 1991; Zilhao et Almeida 2002), précisent le cadre paléoenvironnemental de ces séjours inscrit dans le dernier pléniglaciaire würmien dans ces régions calcaires de moyenne altitude d'Estrémadure. Ces régions étaient alors couvertes d'une végétation rare de type subalpin, avec des bouquets de pins dominants. Un bon analogue se trouve dans les forêts de pins du versant sud des Pyrénées, à une altitude de 1 100-1 $800 \mathrm{~m}$, avec une moyenne annuelle de température de $7-10^{\circ}$ et des précipitations annuelles entre 800 et $1500 \mathrm{~mm}$. Ces conditions en Estrémadure indiquent des conditions bioclimatiques fortement affectées par l'avancée du dernier front glaciaire (OIS 2).

\section{Matériel et contexte}

Le matériel faunique représente près de 1800 restes provenant de cinq couches différenciées à la fouille. Les couches supérieures (c.0) et de base (c.3 et 4) ne livrent que de très rares éléments. La surface excavée est relativement réduite (moins de $10 \mathrm{~m}^{2}$ ) mais correspond à la totalité de la surface occupée par les groupes humains. La majeure partie des vestiges fauniques (tabl.1) est issue de la couche $2(n=1516)$, puis de la couche $1(n=214)$. Ils sont essentiellement constitués de lagomorphes, soit en moyenne $89 \%$ de l'ensemble. Si on exclut les rares éléments intrusifs tels que gastéropodes et petits vertébrés, ce chiffre monte à 94,7\%, le reste correspondant à des Caprinés (5,3\%). L'archéofaune d'Anecrial peut ainsi être considérée comme quasiment monospécifique, provenant de deux niveaux principaux, et relativement peu abondants en fonction de la densité des restes et des individus. Pour ces niveaux, les occupations humaines et l'ensemble du matériel lithique et osseux, sont organisés autour de zones de combustion (foyers) centrées dans les carrés $M-$ $\mathrm{L} 20$ pour le niveau 1 et $\mathrm{J}-\mathrm{K} 21$ pour le niveau 2 ; ces installations se situent près d'une des parois de la cavité (bande J).

L'ensemble des sédiments a été systématiquement tamisé à une maille de $3 \mathrm{~mm}$ et les sédiments du foyer de la couche 2 ont été tamisés à une maille de $1 \mathrm{~mm}$ permettant la récupération des plus petits vestiges (Almeida, comm.pers.). Les ossements de lagomorphes se fracturent facilement et génèrent de nombreuses petites esquilles et débris (fig. 2) ; ceux-ci sont aisément, vu leur gracilité, rapportables à ce petit mammifère. II existe ainsi une grande différence entre le nombre de restes total (NRT) et le nombre d'éléments anatomiques identifiés (NRD=NISP). Pour la couche 1 , le NRD s'élève à $49,5 \%$ sur un nombre total de lagomorphes de 184 restes; pour la couche 2, il est d'environ $39 \%$ sur un nombre total de 1 417 restes. Ces chiffres indiquent non seulement une fracturation anthropique importante mais suppose également une fragmentation post-dépositionnelle en relation avec une compaction et un piétinement ainsi qu'un réajustement synsédimentaire du dépôt formé de cailloutis de petits modules (5-10 cm dans la c. 1$)$ ou de sable calcaire relativement lavé (c.2).

Parmi les plus petits éléments, on note la présence discrète de poissons (trois vertèbres dans la c.2), d'amphibiens (un reste dans la c.2) et d'oiseaux (un et six restes dans les 


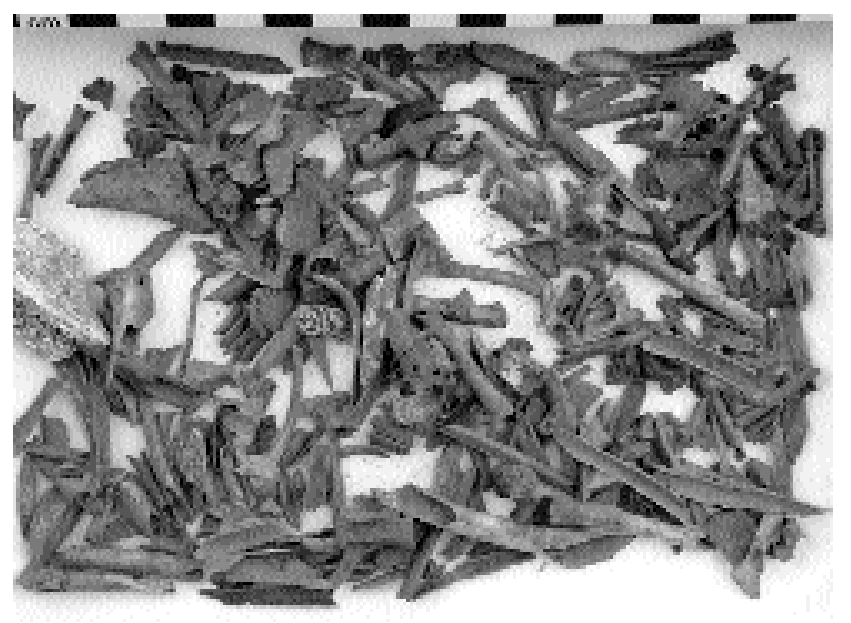

c.1 et 2). La présence de coquilles de gastéropodes terrestres de petite taille est à relever avec une forte proportion dans le niveau le plus superficiel (c. 0$)$ : 60 sujets composés d'au moins deux espèces (dont une de morphologie aplatie) ; ils sont présents sur toute la séquence mais leur nombre diminue vers le bas. Ces divers taxons représentent des constituants du sédiment et leur origine peut être soit naturelle, soit liée à des déjections de prédateurs (rapaces ou petit camivore).

\section{Taphonomie des Assemblages osseux}

\section{Les Capridés}

Les restes d'un mammifère de moyenne taille sont peu nombreux : 15 dans la c. 1 et 75 dans la c. 2 (tabl.2). Les éléments diagnostics sont rares avec un fragment d'extrémité distale d'humérus, un fragment de cavité cotyloïde et une esquille de diaphyse (face postérieure) de métatarse permettant de rapporter ce matériel à un capriné, de la taille de Capra (cf. C. pyrenaica). Aucune dent n'est présente. En
Figure 2 - Matériel de Lagomorphes d'Anecrial ; notez les nombreuses petites esquilles et le matériel noirci (chauffé et brûlé).

Figure 2 - Faunal (lagomorphs) remains from Anecrial; notice the abundance of small splinters and the heated and burnt pieces. regard de la taille générale des autres restes et de leurs conditions physiques (altération, couleur), nous avons rapporté l'ensemble de ce matériel au même genre. Les esquilles sont les plus abondantes et ont une taille moyenne de $37 \mathrm{~mm}$ (de 21 à $86 \mathrm{~mm}$ ). Dans l'ensemble, les bords de cassures indiquent des fragmentations sur os déjà secs (cassures obliques et denticulés, bords mousses, face rugueuse). La couche 1 est particulièrement pauvre et on note quelques pièces portant des traces de feu $(n=4)$. La couche 2 montre de nombreux fragments de côtes, et deux fragments d'épiphyses indiquent un sujet relativement jeune (jeune adulte ?) ; il n'y a aucune trace de combustion sur les ossements de la c.2 et une esquille porte de légères traces de dents d'un camivore de petite taille.

Ce matériel montre un état de conservation similaire entre les deux couches : les ossements sont dégradés, souvent de couleur blanche, avec un degré d'altération relativement marqué (stade 3 selon Behrensmeyer 1978) ; quelques pièces développent un concrétionnement sous la forme d'un voile de calcite (épaisseur maximale de $3 \mathrm{~mm}$ ). De même, les cupules de dissolution sont nombreuses et

\begin{tabular}{|c|c|c|c|c|c|c|}
\hline & c. 1 & c. 2 & $n$ siries & juv & n carrè & Garrè \\
\hline coste & 1 & 26 & 3 & & 4 & L22 \\
\hline Hurnèrus droil & & 1 & 1 & & 1 & L22 \\
\hline Métatarse (toct.disphyse) & & 1 & 1 & & 1 & L20 \\
\hline sésarnoide & 1 & & & & 1 & \\
\hline Pelvis (tgt. cosvite) & & 2 & 1 & & 2 & $\mathrm{~J}-\mathrm{K} 21$ \\
\hline épiphyse (fgl.) & 2 & 2 & & 2 & - & \\
\hline esquille & 4 & 36 & 3 & & 5 & L22 \\
\hline os plat indét. & & 2 & & & - & \\
\hline microesquilles & 7 (dont 4 brules) & 5 & & & 3 & L22 \\
\hline total & 15 & 75 & 9 & 2 & & \\
\hline
\end{tabular}

Tableau 2 - Composition anatomique des restes de Caprinés d'Anecrial (et détail de nombre de stries, juvéniles et localisation pour la c.2). $n$ car $=$ nombre de carré ou l'élément est présent; ca né= carré ou l'élément est majoritaire

Table 2 - Anatomical parts of Caprids from Anecrial (and detail: cut-marks, juvenile and location for level 2). 

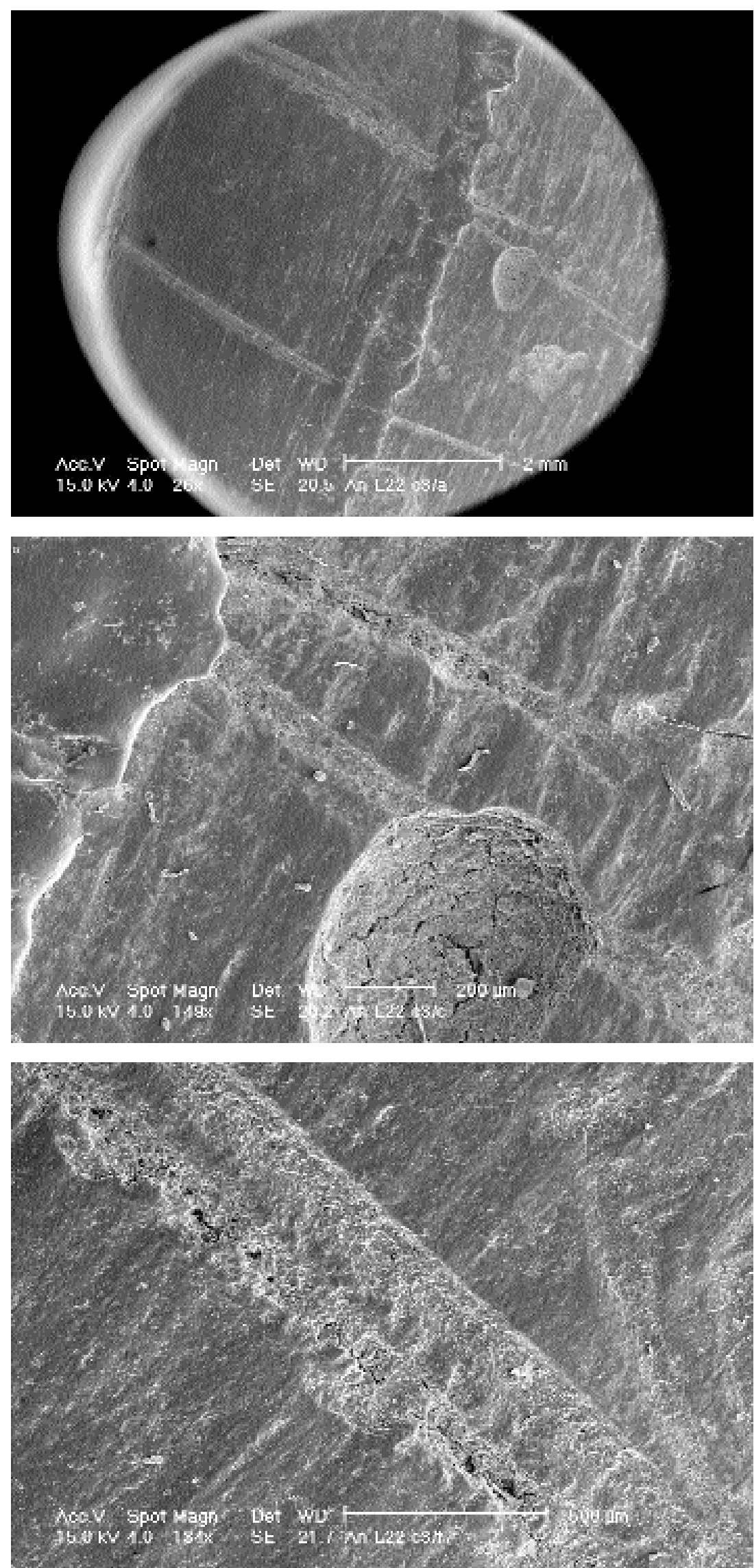

Figure 3 - Strie de découpe sur une esquille de mammifères (capridés) d'Anecrial (L22-C.2) pris au MEB. a- général ;

b- détail d'une cupule de dissolution ;

$c$ - détail d'une strie de découpe montrant des traces marginales dues à des frottements (sur la droite) ; d'après la morphologie de la strie, le geste de l'outil, support retouché, est dirigé vers le haut.

Figure 3 - Cut-marks on bone splinters of Caprids (L22-layer 2), SEM pictures. 
recoupent parfois des traces laissées par des supports lithiques (fig. $3 \mathrm{a}-\mathrm{c}$ ). Les stries de boucherie, généralement courtes, existent à la fois sur des esquilles $(n=3)$, sur des fragments de côtes $(n=3)$ et sur les trois éléments déterminables : une strie sur l'épicondyle de l'humérus, sur la diaphyse du métatarse et autour de la cavité cotyloïde. Ces actions marquent nettement l'exploitation et la consommation par l'homme d'un bouquetin. Les rares éléments déterminables ne désignent que des fragments de la carcasse avec des régions anatomiques différentes (axial, antérieur, postérieur). L'introduction d'une carcasse entière ou en morceaux est alors difficile à préciser.

Ces observations taphonomiques indiquent que ces restes sont restés exposés longtemps sur le sol, subissant non seulement des actions climato-édaphiques mais également des actions mécaniques démontrées par des cassures sur os secs, dues aux piétinements et/ou déplacement des vestiges. De telles actions sur les ossements de lagomorphes entraîneraient certainement des dégâts plus importants sur ces os plus fragiles, dégâts qui ne se reflè tent pas dans les décomptes de cette espèce. La plus forte densité des fragments osseux de caprinés de la couche 2 (carrés K-L/21-22) est décalée de celle des lagomorphes $(\mathrm{J}-\mathrm{K} / 20-21)$ et des artefacts lithiques. On insistera de nouveau sur la différence d'état de conservation entre les deux espèces avec les restes de caprinés beaucoup plus altérés. La taille et la condition des esquilles de ce taxon, l'absence de petits débris d'épiphyses, ne permettent pas non plus de soutenir l'hypothèse d'une utilisation pour la formation de bouillon graisseux (Zilhao 1997), comme cela a été avancé par exemple au Flageolet I (Bézenac, Dordogne), niveau du Périgordien supérieur (Delpech et Rigaud 1974).

Sur la base de ces critères, il est possible de soutenir que les restes de bouquetin (un seul individu) sont issus d'une occupation humaine plus ancienne, antérieure à celle livrant des lagomorphes associés aux foyers et à la majorité des vestiges lithiques. Quelques fragments osseux de ce rupicole auraient ensuite été remobilisés dans ce dépôt légèrement en pente pour se mélanger aux deux installations postérieures (c. 2 et 1). L'occupation de la couche 1 est très ponctuelle avec un faible investissement (peu de lithique, peu de faune) ; elle pourrait désigner un exemple de séjour paticulièrement bref, comme celui qui a pu se faire lors de cette phase plus ancienne de présence humaine dans la cavité. Une autre explication suggérait l'introduction de morceaux de bouquetin (Zilhao 1997), viande avec fragments osseux transportés lors de déplacements du groupe et représentant des vivres de voyage. L'état plus altéré des restes osseux, l'absence d'éléments crâniens et de dents, pourraient alors correspondre à un aliment (sensu carcasse) ayant subi une préparation culinaire (sélection des morceaux, découpe) en vue de stockage puis d'une consommation différée. Ce comportement se serait alors manifesté aussi bien chez les solutréens (c.1) que chez les gravet- tiens (c.2). Au vu de nos analyses, et des résultats radiométriques obtenus sur un os de capriné, cette hypothèse peut également être écartée.

\section{Les Lagomorphes (Famille des Leporidae)}

Les restes de lagomorphes ont été retrouvés principalement dans et autour de zones de combustion importante, localisées différemment dans les deux principaux niveaux. Le foyer de la couche 2 est plus proche de la paroi que celui de la couche 1. L'ensemble des pièces est attribué au lapin de garenne, Oryctolagus cuniculus ${ }^{4}$, avec près de 1600 restes dont environ $40 \%$ réellement déterminables anatomiquement et taxonomiquement. Au total, ils représentent au moins 327 éléments (NME), soit une estimation de 19 individus (quatre pour la couche $1 ; 15$ pour la couche 2). II s'agit ici d'ensembles que l'on peut qualifier de 'pauvres' par comparaison avec certains sites préhistoriques livrant des dizaines de milliers d'éléments de léporidés concernant des centaines d'animaux.

Les ensembles d'Anecrial reposent sur un faible nombre de restes et d'individus, en particulier pour la couche 1. Le nombre minimal d'individus (NMI) est identique entre les estimations faites par fréquence ou par combinaison ; de même, les fréquences relatives utilisant le NMI ou le MAU sont également similaires et interchangeables (fig.4). Le tableau 3 détaille les compositions anatomiques et les obsenations taphonomiques ; les débris sont particulièrement nombreux (esquilles et microesquilles : ces demières inférieures à $1 \mathrm{~cm}$ ) et proviennent principalement d'os longs des membres (humérus, radius, fémur, tibia).

Globalement, toutes les parties du squelette sont présentes mais les pourcentages entre éléments montrent des disparités intéressantes. De plus, il y a un certain équilibre entre ossements droits et gauches, bien que de nombreux fragments n'aient pu être latéralisés. Ceci suppose l'apport entier de carcasses dans la cavité. Le matériel est en bon état de conservation et les surfaces osseuses sont très peu altérées (au moins macroscopiquement). Environ $6 \%$ (c.1) à 1\% (c.2) des restes présentent des traces de comosion avec des cupules de dissolution dues à des actions chimiques, certaines en relation avec le développement de radicelles ou de mousses (lichens) (fig. $5 \mathrm{a}-\mathrm{b}$ ) : cette altération affecte d'ailleurs les plus grosses pièces et développe des zones sombres, pouvant être confondues avec l'action de flammes. L'altération est bien visible sur les mandibules avec des perforations dans les zones d'os peu épais comme le diastème ou à la base de la troisième prémolaire, face linguale. Par ailleurs, un léger concrétionnement est observable sur environ $6 \%$ (c.1) à moins d' $1 \%$ (c.2) des restes, nettement plus faible que celui constaté sur les pièces de Caprinés. Un autre exemple, plus anecdotique, affecte la surface d'un fémur gauche (K21-c.2-85) avec la présence de cinq sillons bien limités et flanqués de fins reliefs (fig. $5 \mathrm{c}-\mathrm{e}$ ). Ils sont interprétés comme des

(4) On distingue principalement deux sous-espèces de lapin en Europe : O.c.cuniculus (Linné 1758) présente au Nord et O.c.huxleyi (Haeckel 1874) occupant le pourtour méditerranéen et les îles, de plus petite taille (Miller 1912 in SéronieVivien 1994). Les faibles dimensions des spécimens d'Anecrial les rapprochent de cette dem iè resous-espèce. 


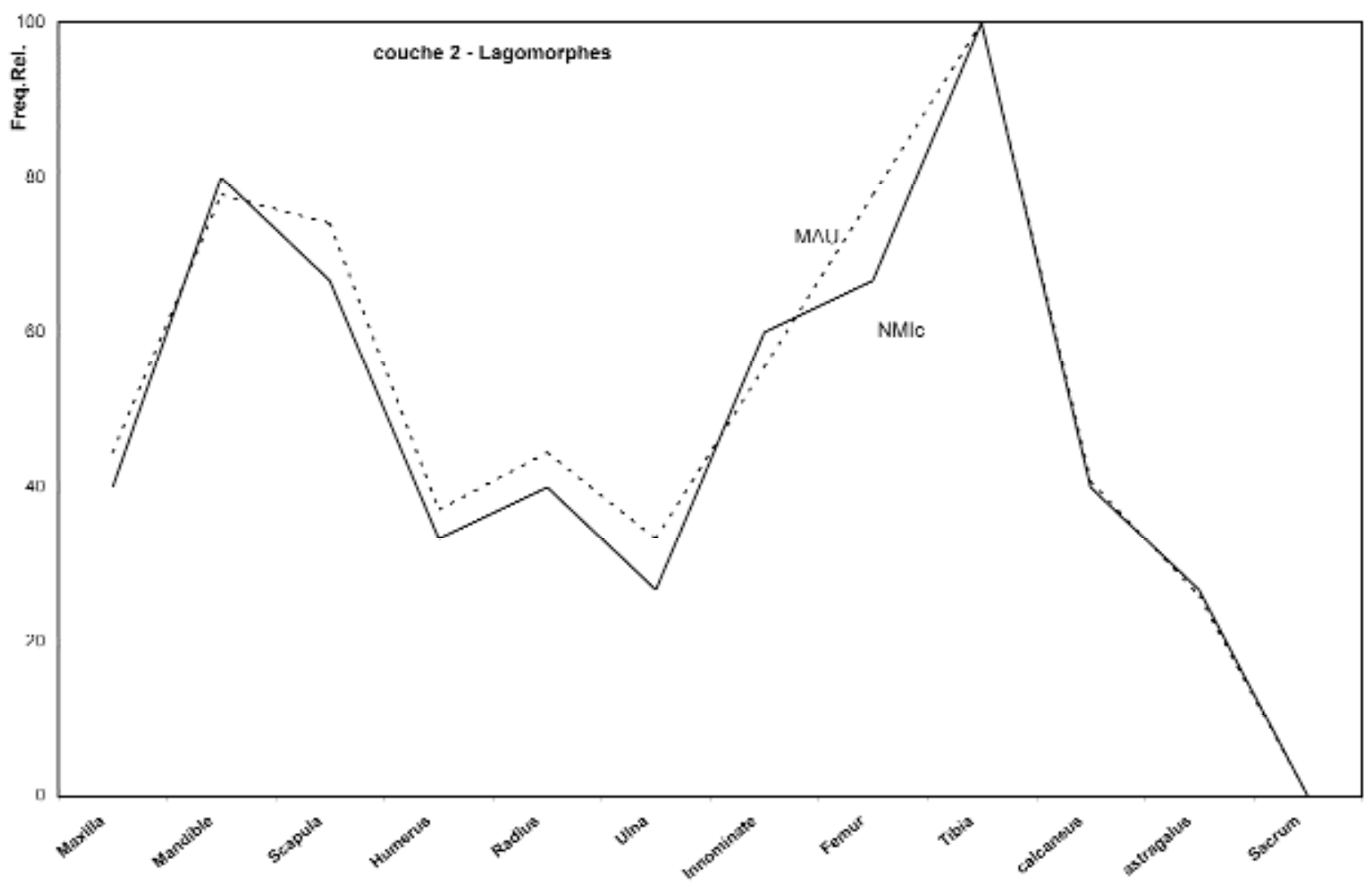

Figure 4 - Représentations squelettiques des lagomorphes (c.2) suivant le MAU et le NMIc.

Figure 4 - Lagomorph's skeletal representation (layer 2) in MAU and MNIc.

traces laissées par la radula de gastéropodes. Ceux-ci sont connus pour attaquer les ossements de petite taille (Morel 1986), bien que les traces observées ici soient différentes de celles rapportées par cet auteur. Un métapode entier de la c. 2 montre des micro-perforations pouvant comespondre à l'action de vers, impliquant la présence de tissus mous. Ces détails permettent de supposer une exposition avant enfouissement, laissant ces organismes agir, mais de courte durée en raison de la bonne conservation générale. L'observation à la binoculaire (sur les os longs les plus fréquents) laisse apparaître des striations pouvant être dues à des piétinements, compaction ou déplacement mineur des objets.

Les restes de jeunes sont relativement faibles avec un jeune et un jeune adulte pour la couche 1 (seulement cinq restes sur 184), et au moins deux juvéniles pour la couche 2 (soit 14 restes sur 1417 ; environ $1 \%$ en NRD et environ $13 \%$ en NMI). Les lapins ont un fort taux de reproduction avec généralement deux pics de mise bas, un au printemps et l'autre en automne (cf. Hockett et Bicho 2000 : 721). Cette saisonnalité est toutefois variable et les conditions générales des milieux (climat, alimentation, prédation) constituent autant de facteurs qui modifient ces rythmes biologiques. La mortalité des juvéniles est en général importante pendant les trois premiers mois, et c'est vers 9 mois que l'animal devient adulte. A Anecrial, les adultes sont donc largement majoritaires (au moins pour la c.2) suggérant que les fréquentations du site se placent en dehors de pics reproductifs (soit été ou hiver) ; il reste néanmoins difficile d'affirmer des saisons sur la base d'assemblages de léporidés. Le faible nombre d'individus, associé aux autres données contextuelles, implique des occupations de courte durée, très vraisemblablement intra-saisonnière, qu'il est possible, dans un premier temps, de mettre en relation avec des périodes de mobilité plus grande de la part de groupes humains de taille réduite. De tels groupes sont susceptibles de se former durant certaines saisons, en particulier lors des 'bonnes' saisons (printemps-été), lorsqu'on assiste à une plus grande dispersion des ressources animales.

Les traces de combustion sont relativement peu nombreuses, plus fréquentes sur les fragments. Pour la c.2, près de $17 \%$ (dont $13 \%$ sur des pièces déterminables) montrent une carbonisation (couleur noire) avec quelques pièces de couleur brun-foncée qui auraient subies une crémation atténuée, avec des surfaces desquamées ; il n'y a 


\begin{tabular}{|c|c|c|c|c|c|c|c|c|c|c|c|c|}
\hline couche 1 & G & D & indét & NISP & NME & NMIr & NMle & $\mathrm{j} / \mathrm{j} \mathrm{ad}$ & chaur & brul & n car & carre \\
\hline fol crane & & & 2 & 2 & & 1 & 1 & & 1 & & 1 & \\
\hline Mexillsire & & & & 0 & 0 & 0 & 0 & & & & & \\
\hline Pré-naxillaire & & & & 0 & 0 & 0 & 0 & & & & & \\
\hline Maridfoule & 1 & 1 & 0 & 2 & 2 & 1 & 1 & & & & 1 & - \\
\hline dente isolteses & & & 10 & 10 & & - & & & 1 & 1 & 2 & M21 \\
\hline Incisives & & & 4 & 4 & & - & & & & & 4 & - \\
\hline Vertebres & & & & 0 & 0 & & & & & & & \\
\hline cóles & & & 4 & 4 & 3 & 1 & 1 & & & & 3 & - \\
\hline Scapula & 1 & 0 & 4 & 5 & 3 & 1 & 2 & & & 1 & 3 & - \\
\hline Hurnérus & 2 & 2 & 3 & 7 & 6 & 2 & 3 & & 2 & 1 & 4 & M21 \\
\hline Redius & 1 & 0 & 4 & 5 & 5 & 1 & 3 & & & & 3 & - \\
\hline Ulna & & & 1 & 1 & 1 & 1 & 1 & & & & 1 & - \\
\hline Pelvis & 2 & 1 & 1 & 4 & 3 & 2 & 2 & & & & 3 & $M 21$ \\
\hline Fentrui & 3 & 4 & 2 & 9 & 8 & 3 & 4 & $0 / 1$ & & & 4 & M21 \\
\hline $\begin{array}{l}\text { Trisis } \\
\text { Rolule }\end{array}$ & 3 & 2 & 3 & 8 & 8 & 3 & 4 & & 2 & & 3 & $M 21$ \\
\hline Colcaneum & 0 & 2 & 0 & 2 & 2 & 2 & 2 & $1 / 1$ & & & 2 & - \\
\hline Talue & & & & 0 & 0 & 0 & 0 & & & & & \\
\hline Tarsiens & & & 1 & 1 & 1 & 1 & 1 & & & 1 & 1 & - \\
\hline Melapode & & & 16 & 16 & 11 & - & & $1 / 0$ & & 1 & 4 & M21 \\
\hline Pholange I & & & 10 & 10 & 8 & - & & & & & 5 & M21 \\
\hline Phislange II & & & 1 & 1 & 1 & - & & & & & 1 & - \\
\hline Phislange III & & & & & & & & & & & & \\
\hline esquile & & & 13 & 13 & & & & 1 & & & 4 & $M 21$ \\
\hline microesquille & & & 80 & 80 & & & & & 3 & 6 & 5 & M21 \\
\hline Total & 13 & 12 & 159 & 184 & 62 & 3 & 4 & $3 / 2$ & 9 & 11 & 5 & \\
\hline couche 2 & & & & & & & & & & & & \\
\hline fgt crano & & & 60 & 60 & & - & 0.7 & & & 3 & 6 & $K O D$ \\
\hline Marilaire & 5 & 5 & 5 & 15 & 10 & 5 & 6 & & & 1 & 4 & $\mathrm{~K} 21$ \\
\hline Prto-maxillaire & 7 & 5 & 1 & 13 & 12 & 7 & 7 & & & & 6 & $\mathrm{KOD}$ \\
\hline Mandibuls & 12 & 8 & 6 & 26 & 21 & 12 & 12 & & & 1 & 5 & $\mathrm{~K} \geq 1$ \\
\hline donts igolitos & & & 50 & 50 & & & & & & 1 & 6 & 121 \\
\hline Incisives & & & 28 & 28 & & & & & & 1 & 7 & $K 20-21$ \\
\hline Vertibros & & & 26 & 26 & 12 & - & & 200 & & 10 & 5 & KO1 \\
\hline cotes & & & 60 & 60 & 650 & - & & & 1 & & $\mathrm{~B}$ & 121 \\
\hline Scapula & 10 & 10 & 3 & 23 & 20 & 10 & 10 & & & 3 & 5 & $K 20-21$ \\
\hline Humbrus & 2 & 2 & 14 & 18 & 10 & 2 & 5 & $1 \%$ & & 1 & 6 & K21 \\
\hline Radius & 4 & 4 & 17 & 25 & 12 & 4 & 6 & 100 & & 1 & 6 & 121 \\
\hline Uina & 3 & 2 & 12 & 17 & 9 & 3 & 4 & & & 2 & 7 & - \\
\hline Polvis & 6 & 4 & 12 & 22 & 15 & 6 & 9 & & & 3 & 9 & 121 \\
\hline Femar & 6 & 7 & 29 & 42 & 21 & 7 & 10 & 30 & & 1 & B & $K \geq 1$ \\
\hline Tibia & 14 & 15 & 13 & 42 & 27 & 15 & 15 & 30 & & 1 & 7 & K21 \\
\hline Rotule & & & 1 & 1 & 1 & 1 & 1 & & & & 1 & - \\
\hline calcanoum & 6 & 4 & 1 & 11 & 11 & 6 & 6 & 1,10 & & 2 & 4 & $\mathrm{~K} 21$ \\
\hline Talus & 2 & 4 & 1 & 7 & 7 & 4 & 4 & & & 1 & 4 & KQ1 \\
\hline Tarsions & & & 1 & 1 & 1 & 1 & 1 & & & & 1 & \\
\hline Motapode & & & 50 & 50 & 15 & - & & 300 & 1 & & 9 & $K 20-21$ \\
\hline Phalange I & & & 11 & 11 & 11 & - & & & & & 5 & $K \geq 0$ \\
\hline Phalango if & & & 4 & 4 & & & & & & & 3 & - \\
\hline Fhalango III & & & 1 & 1 & & & & & & & 1 & - \\
\hline copuilis & & & $5 \notin 5$ & 5465 & & & & & & 111 & 6 & $\mathrm{KO1}$ \\
\hline microcsquilio & & & 299 & 299 & & & & & & 94 & $B$ & $\mathbb{2 1}$ \\
\hline Total & 77 & 71 & 1270 & 1417 & 215 & 15 & 15 & 140 & 2 & 237 & 9 & \\
\hline
\end{tabular}

Tableau 3 - Composition anatomique et observations taphonomiques des Lagomorphes d'Anecrial. $G=g a u c h e ; D=d r o i t$, j=juvénile ; jad=jeune adulte ; Chauf=chauffé (faible action des flammes : couleur brunâtre) ; brul=brûlé (carbonisé) ; $n$ car= nombre de carré ou l'élément est présent ; carré= carré ou l'élément est majoritaire.

Table 3 - Anatomical parts and taphonomic observations of Lagomorphs from Anecrial. 

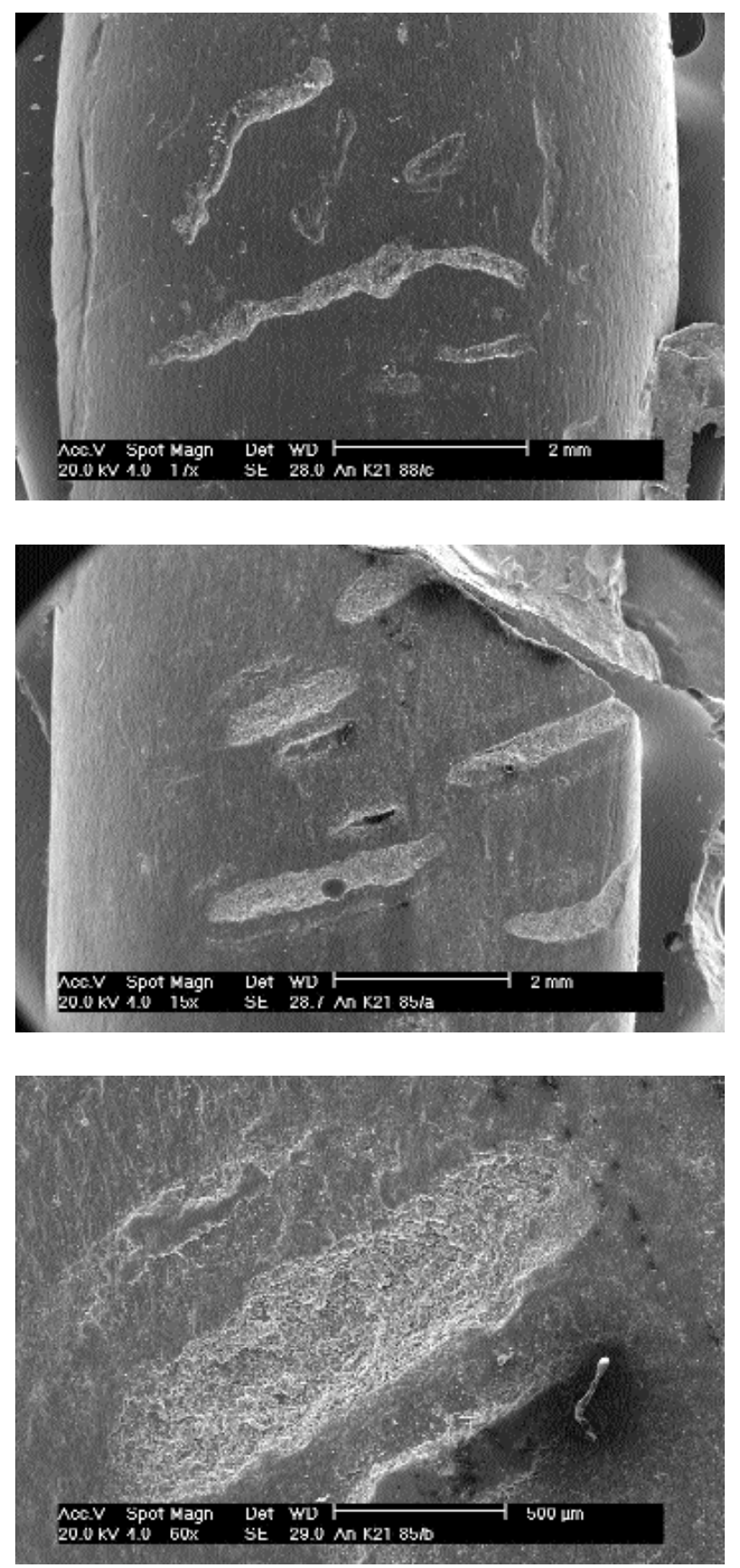

pas de pièces calcinées (gris-blanc). La combustion touche toutes les pièces anatomiques, depuis des éléments crâniens (et des dents isolées), des vertèbres et des os longs ou courts. La crémation reste donc limitée avec une localisation majoritaire des vestiges sur les bords du foyer, indiquant cependant un rejet systématique vers celui-ci (destruction totale de certaines éléments). D'autres actions anthropiques sont plus discrètes, seulement visible en c.2 : une strie de découpe sur un métapode entier désignant une activité de dépouillage, et quatre fragments de pelvis (avec l'acétabulum) montrant des sillons
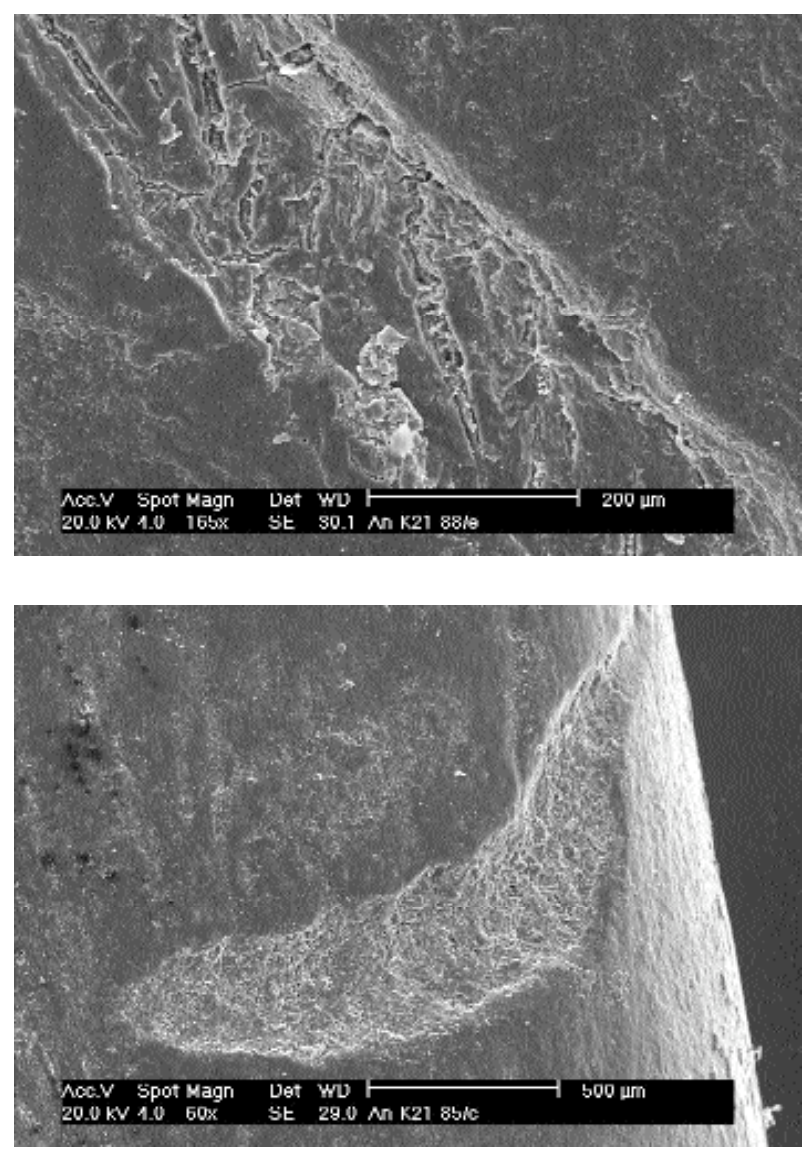

Figure 5 -

A - Surface de fémur de Lagomorphes (K21-c.2-88) montrant des traces de radicelles : a- général ; $b$-détail interne de sillons ; $B$ - Surface de fémur de Lagomorphes (K21-c.2-85) montrant l'action de gastéropodes : c- général ; $d$ et e-détails

\section{Figure 5 -}

A- lagomorph's femur surface (K21-2-88) with root-marks a-detail, $b$-internal detail of grooves);

$B$ - lagomorph's femur surface (K21-2-85) with gastropod gouging (c-general and d,e- details)

relativement superficiels, larges, attribués hypothétiquement à des marques de dents humaines (voir à ce sujet Perez Ripoll 2004). Globalement il n'y a pas de perforations attribuables à des petits camivores ou à des coups de becs de rapaces, souvent localisées sur les extrémités d'os longs (ex. in Cochard 2004). II faut cependant noter que les extrémités sont faiblement présentes dans ces ensembles (cf. infra). Une exception concerne un pelvis (L21-c.2-6) présentant une perforation (puncture) près de la cavité cotyloïde (diamètre de $1 \mathrm{~mm}$ ) attribuée à un carnassier de petite taille. 
Couche 1

\section{ELEMENT}

Maxilla

Mandible

Scapula

Humerus

Radius

Uina

Innominate

Femur

Tibia

calcaneus

astragalus

Sacrum
NISP MNE MAU MAU rieq.Re

$$
\begin{gathered}
0,00 \\
25,00 \\
37,50 \\
75,00 \\
62,50 \\
12,50 \\
37,50 \\
100,00 \\
100,00 \\
25,00 \\
0,00 \\
0,00
\end{gathered}
$$

Couche 2

\section{ELEMENT}

\section{Maxilla}

Mandible

Scapula

Humerus

Radius

Ulna

Innominate

Femur

Tibia

calcaneus

astragalus

Sacrum
NMlc NMlc rres-Rel.

$$
0,00
$$

50,00

75,00

75,00

25,00

50,00

100,00

100,00

50,00

0,00

0,00
Tableau 4 - Synthèse des données sur les lagomorphes d'Anecrial.

NISP = nombre de spécimens identifiés ;

$N M E=$ nombre minimal d'éléments ;

$M A U=$ unité animale minimale ;

NMIc = nombre minimal d'individus (par combinaison);

Freq. Rel. = fréquence relative.

Table 4 - Synthesis of data on Lagomorphs from Anecrial.

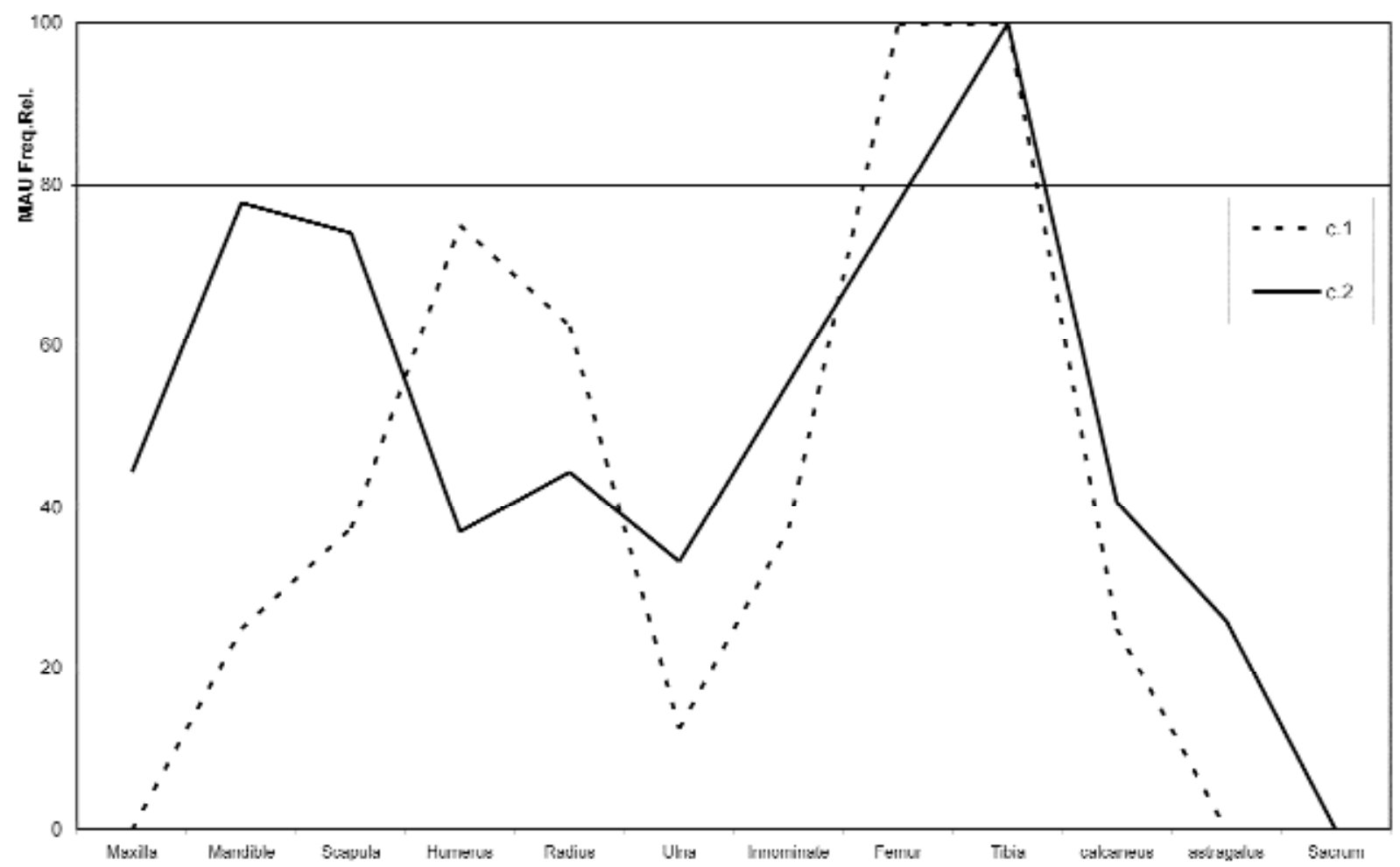

Figure 6 - Représentations squelettiques des lagomorphes : comparaison entre les couches suivant la fréquence relative du MAU.

Figure 6 - Skeletal representation of Lagomorphs: relative frequency (based on MAU) comparison between the two layers. 


\begin{tabular}{|c|c|c|}
\hline couches & 1 & 2 \\
\hline Scapula avcc cavité & 3 & 19 \\
\hline Scapula diaphyse & 2 & 4 \\
\hline Humcrus enticr & 0 & 0 \\
\hline Humérus proximal & 0 & 3 \\
\hline Humćrus distal & 4 & 1 \\
\hline Humèrus cylindre & 3 & 11 \\
\hline Radius enticr & 0 & 0 \\
\hline Radius proximal & 0 & 4 \\
\hline Radius distal & 0 & 3 \\
\hline Radius cylindre & 5 & 18 \\
\hline Ulna cntior & 0 & 0 \\
\hline Ulna proximal & 1 & 5 \\
\hline Ulna distal & 0 & 0 \\
\hline Ulna cylindre & 0 & 12 \\
\hline Pelvis avec cavitè & 4 & 15 \\
\hline Polvis branche & 0 & 6 \\
\hline Fèmur entier & 0 & 0 \\
\hline Fémur proximal & 0 & 1 \\
\hline Fèmur distal & 1 & 2 \\
\hline Fémur cylindre & 5 & 23 \\
\hline Tibia entier & 0 & 0 \\
\hline Tibia proximal & 0 & 4 \\
\hline Tibia distal & 4 & 10 \\
\hline Tibia cylindre & 4 & 24 \\
\hline Calcaneum entier & 2 & 9 \\
\hline Calcaneum fragment & 0 & 2 \\
\hline Talus entier & 0 & 6 \\
\hline Talus fragment & 0 & 1 \\
\hline Métapode entier & 5 & 9 \\
\hline Metapode proximal & 6 & 10 \\
\hline Métapode distal & 2 & 11 \\
\hline Mètapode cylindre & 0 & 19 \\
\hline Phalanges entières & 7 & 14 \\
\hline Phalanges fragments & 2 & 2 \\
\hline total & 60 & 248 \\
\hline
\end{tabular}

Tableau 5 - Détail des représentations squelettiques des Lagomorphes d'Anecrial (NRD).

Table 5 - Skeletal parts representation (detail) of Lagomorphs from Anecrial (NISP).

Tableau 6 - Fréquences (\%NRD) des extrémités vs. cylindres et de la longueur

Couche 2

Extrérnités Cylindress

\% cylindre

\begin{tabular}{lcccc|} 
& Extrèrnités & Cylindres & \multicolumn{2}{c|}{ \% cylindre } \\
& & & $\times 1 / 2$ (long) & $=1 / 2$ (court) \\
\hline Hurnèrus & 26,67 & 73,33 & 72,73 & 27,27 \\
Radius & 28 & 72 & 44,44 & 55,56 \\
Ulna & 29,4 & 70,6 & 16,67 & 83,33 \\
Fémur & 11,54 & 88,46 & 69,57 & 30,43 \\
Tibja & 36,84 & 63,16 & 79,17 & 20,83 \\
Metapodes & $61,23^{*}$ & 38,77 & & \\
\hline
\end{tabular}

*Mpp entier inclut des cylindres, pour les principaux os longs de Lagomorphes d'Anecrial (c.2).

Table 6 - Frequency (\%NISP) of ends vs cylinders and length of cylinders, for the main long bones of Lagomorphs from Anecrial (layer 2). 

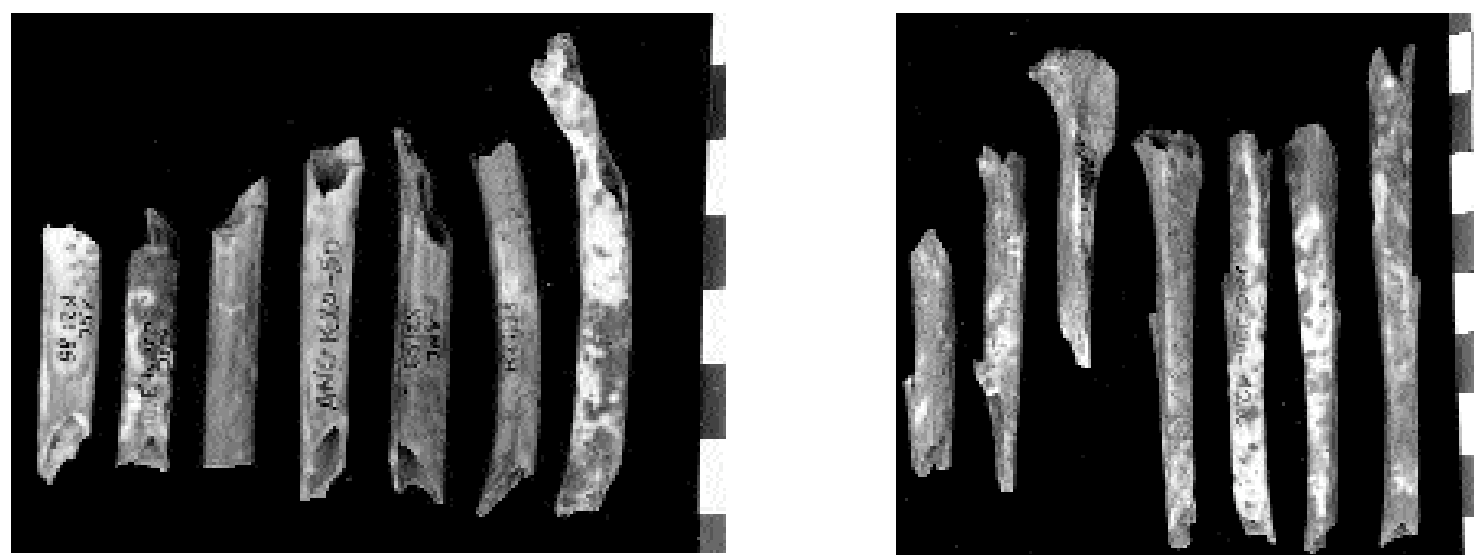

Figure 7 - Cylindres et portions de fémur (a) et de tibia (b) de lapins d'Anecrial (en a, à gauche, portion diaphysaire de fémur avec traces de gastéropodes : cf. fig. $4 B)$.

Figure 7 - Cylinders and parts of femur (a) and tibia (b) of the rabbit from Anecrial (the extreme left femur specimen is the one shown in fig.4B).

Comme il a été noté, tous les éléments du squelette sont présents mais le squelette axial (vertèbres, cotes) et les parties distales des membres (tarsiens, carpiens, métapode et phalanges) sont nettement sous-représentés, ne pouvant s'expliquer ni par une conservation différentielle ni par une collecte sélective à la fouille. Le squelette céphalique (mandibules, fragments crâniens, dents isolées) et le squelette appendiculaire (os longs des membres) sont majoritaires. Les profils de représentation (tabl.4 et fig. 6) sont assez comparables entre les deux couches, avec cependant une meilleure fréquence des parties crâniennes (mandibules et maxillaires) dans la couche 2 ; et, dans une moindre mesure, une représentation plus grande des éléments antérieurs (humérus, radius) dans la couche 1. Ceci pourrait être mis en relation avec la plus faible occupation de la couche 1 ou bien par une différence de technique de consommation («écrasement» des crânes pour récupérer le cerveau ?).

Les os longs du membre postérieur sont aussi abondants en nombre de restes que ceux du membre antérieur, mais ces derniers montrent de plus faible fréquence relative, en rapport avec leur plus grande fragilité. Ce sont les tibias, suivis par les fémurs, qui fournissent le plus d'éléments et donc le NMI (plus la mandibule bien représentée aussi dans la couche 2). Les décomptes sont détaillés dans les tableaux 5 (détail anatomique) et 6 (couche 2 : rapport extrémités/diaphyse et longueur des cylindres).

A l'exception des métapodes, il n'existe pas d'os longs entiers et ce sont les portions diaphysaires, ou cylindres, qui dominent (73,3\% en moyenne). Si l'humérus, le fémur et le tibia possèdent des cavités médullaires importantes et donc plus riches en moelle, ce n'est pas le cas pour le radius et l'ulna. Le manque d'extrémités ne peut s'expliquer par des questions de conservation et supposerait alors soit un rejet hors de la zone considérée ou bien une consommation particulière, notamment pour celles plus riches en os spongieux (humérus proximal, fémur proximal et distal, tibia proximal). Pour le membre antérieur, le pourcentage d'extrémités est comparable entre les différents éléments. Le fémur montre la plus faible fréquence en extrémités et le tibia la plus forte avec effectivement très peu d'extrémité proximale. II faut reconnaître que nos effectifs sont réduits, limitant ainsi l'analyse. Ce manque d'épiphyses est notable ; elles auraient pu être détruites soit par les hommes, soit aussi par un charognard (type renard) passant peu après les installations humaines. Cette dernière activité n'aurait également pas épargné les vertèbres (voir ci-dessous). Les cylindres les plus longs concement l'humérus, le fémur et le tibia (fig. $7 \mathrm{a}$-b) alors que les plus courts sont le radius et surtout l'ulna : ce dernier os est fin et fragile et sa réduction lors de la fracturation est logiquement plus forte bien que leur intérêt alimentaire soit faible voire nul. La présence de cylindres longs dénote de nouveau une faible perturbation postdépôt et l'ensemble peut alors être considéré comme étant en position primaire. Enfin, on notera la bonne représentation des pré-maxillaires, l'absence fréquente des branches montantes des mandibules et la présence de nombreuses dents isolées. Ce dernier point peut de nouveau être relié à un certain temps d'exposition avant enfouissement définitif ${ }^{5}$.

La localisation des restes de lagomorphes est homogène et on ne constate pas de variation suivant les éléments anatomiques (cf. indications in tabl. 3). La couche 1 montre une plus grande densité dans le carré M21, alors que la couche 2 montre des densités en K20-21, démontrant un décalage entre les occupations, situées plus contre la paroi pour la couche 2 . On rappellera que le foyer est à cheval entre les carrés $\mathrm{K}$ et $\mathrm{J} 21$ dans cette couche, alors

(5) Le déchaussement des dents de léporidés semble être un phénomène particulièrement rapide, dés lors que le crâne devient sec et sans composant organique (peau, viande, etc.). 
qu'il est plus écarté pour la couche 1, en L20-21 et M21. Ces observations permettent aussi de démontrer la synchronicité, et donc la brièveté, des occupations pour chaque couche.

\section{Comparaison et discussion}

La présence de lagomorphes découverts en contexte archéologique pose toujours la question de leur origine car de nombreux processus peuvent induire de telles accumulations dans les sédiments en général meubles. Le plus souvent évoqué pour le lapin, notamment dans les sites les plus anciens, désigne des mortalités naturelles dans des lieux de vie et de reproduction (terriers : garenne). Un autre processus naturel concerne le résultat d'un piégeage dans une cavité (de type aven par exemple). Les facteurs de prédation sont également responsables d'accumulations relativement importantes; parmi les camivores, le lynx pardelle et le renard sont connus pour exercer une pression de chasse importante sur les léporidés, et leurs fèces contiennent des restes que l'on retrouve dans les tanières ou repaires, outre le transport de parties de carcasses. Le lynx en particulier, possède une alimentation majoritairement orientée vers l'acquisition de lapins: prés de $88,4 \%$ d'après l'examen d'excréments du lynx pardelle (Delibes 1987: 37). D'autres camivores, depuis la belette jusqu'au loup, consomment régulièrement des lagomorphes. Renard et lynx sont justement des espèces très bien représentées dans les faunes actuelles et fossiles (dernier glaciaire) de la péninsule ibérique. Les grands rapaces diurnes (aigles, vautours, buses) ou noctumes (chouettes, hiboux) peuvent également être à l'origine de stocks osseux accumulés près de leurs aires à partir de pelotes de régurgitation (par exemple, Chaline 1974 ; Andrews 1990).

Afin de comprendre les mécanismes de formation des assemblages de petits mammifères, en particulier des lagomorphes, de nombreux travaux ont récemment été effectués à partir de données actuelles avec des applications aux ensembles fossiles. Les sites naturels sont encore peu prospectés (Cochard 2000, 2004) alors que les données sur les aires et les pelotes des rapaces (Cruz-Uribe et Klein 1998 ; Hockett 1989, 1991, 1995, 1996) ou provenant de petits carnivores (Andrews et Nesbit-Evans 1983 ; Cochard 1999 ; Schmitt et Juel 1994 ; Stiner 1994 ; Hockett 1999 ; Sanchis Serra 2000) font l'objet d'une abondante littérature. De même, il existe de nombreux exemples à partir de gisements archéologiques (Vigne et Marinval-Vigne 1983 ; Perez Ripoll 1993, 2004 ; Shaffer 1992 ; Charles et Jacobi 1994 ; Quirth-Booth et Cruz-Uribe 1997 ; Fontana 1998, 1999, 2004 ; Hockett 1994 ; Cochard 2004), dont certains issus de sites portugais (Rowley-Conwy 1992 ; Valente 2000 ; Bicho et al. 2000 ; Hockett et Bicho 2000 ; Hockett et Haws 2002), et d'exemples ethnographiques (par exemple, Yellen 1991; Lupo et Schmitt 2002).

Sans prétendre citer l'ensemble des études taphonomiques sur ce thème, une synthèse a été proposée par Hockett et Haws (2002 : tabl.II). Elle permet de définir des critères pertinents pour reconnaitre l'origine des accumulations de léporidés, portant notamment sur la représentation des parties du squelette et les types de fractures, la structure d'âge des populations et la présence de marques (corrosion, perforations, stries de découpe, brûlures), auxquels on pourrait ajouter la distribution spatiale des vestiges et l'existence de connexions articulaires. L'analyse des deux ensembles de lapins découverts à Anecrial rend compte de l'origine anthropique de ces accumulations. La forte présence de cylindres, dont l'importance est bien mise en évidence par les travaux de M. Perez Ripoll et B. Hockett, le pourcentage d'ossements brûlés et la majorité de sujets adultes confirment cette interprétation. On peut alors avancer pour Anecrial, d'une stratégie de capture de la part des préhistoriques dans les environnements immédiats du gisement.

Le lapin est une espèce relativement grégaire, organisée en groupes familiaux territoriaux couvrant environ 10 à 20 hectares. Ils creusent des terriers complexes (multiples issues) dans les sédiments meubles et leur activité alimentaire se situe à l'aube et au crépuscule. L'ouïe est très développée alors que l'odorat l'est moins et que la vue est médiocre. Cette organisation sédentaire et communautaire s'oppose à celle rencontrée chez le lièvre par exemple, et il est parfois relevé que les deux espèces co-existent mal. Sans l'aide de chien (ou de fouine) pour débusquer et faire courir l'animal, ou d'armes à longue distance, la prise la plus classique de lapins est le piégeage aux abords des terriers ou sur les passées. II est fort vraisemblable que cette technique était connue de la part des préhistoriques qui ont accumulé parfois de grandes quantités de restes de léporidés, comme dans le gisement magdalénien portugais de Picareiro (Hockett et Bicho 2000 : plus de 10000 restes), proche du site d'Anecrial. De tels exemples impliquent non seulement des biomasses appréciables (abondance et régularité de la ressource) mais aussi de plus longues durées d'occupations humaines.

Une recension de la littérature (cf.supra) sur l'abondance des restes de lagomorphes dans les sites fossiles à actuels (genres Lepus, Oryctolagus, Sylvilagus) laisse apparaître au moins deux larges catégories quantitatives (fondées sur le NISP) (Cochard 2004). Le premier concerne des sites possédant un nombre limité de restes (de l'ordre de 15002000 ) ; il s'agit principalement de sites naturels ou formés par des prédateurs non-humains, mais aussi issus d'activités anthropiques. Le gisement d'Anecrial rentre justement dans ce groupe. Les sites contenant plus de 2000 restes correspondent tous à des sites archéologiques : subactuels nord-américains (ex. Hogup cave et 26 NY3393 : Hockett 1995 et 1994 ; Wupatki et Winona : Quirth-Booth et Cruz-Uribe 1997) et du Paléolithique supérieur sud-européen (ex. Picareiro : Hockett et Bicho 2000 ; Gazel : Fontana 1998 ; Pegourié : Séronie-Vivien 1994). Ces derniers désignent des gisements de la fin du Paléolithique supérieur (Magdalénien final) et de l'EpipaléolithiqueMésolithique (Azilien, Sauveterroïde). Auparavant, les restes de ce petit gibier ne sont jamais abondants dans les archéofaunes. Dans le sud de la France par exemple, on constate une différence importante entre gisements du Magdalénien livrant d'important stocks de léporidé et les sites des cultures antérieures où ce petit gibier est faible, 
voire absents (Cochard et Brugal 2004 : fig.1), impliquant des changements majeurs dans les structures techno-économiques et sociales des groupes humains. En outre, il semble que la fin du dernier glaciaire, contemporain de l'expansion des zones forestières, voit diminuer l'exploitation des lagomorphes (Aura et al. 1998 ; Bridault et Chaix 1995) bien que la diversité des ressources, notamment de petite taille (mollusques, poissons, oiseaux, etc.) se poursuive (Bridault 1997). II est cependant fort probable que des particularités régionales apportent quelques nuances à ces schémas généraux ; ainsi, dans la péninsule ibérique, l'exploitation de lapins est plus précoce (Paléolithique supérieur ancien) mais ne semble pas également se poursuivre au début de l'Holocène.

Les gisements abondants en léporidés livrent soit le genre Lepus soit, comme souvent dans la péninsule ibérique, le genre Oryctolagus. L'écologie et l'éthologie de ces deux formes sont suffisamment différentes pour déterminer des stratégies d'acquisitions différenciées. Il est généralement admis qu'une grande quantité de lagomorphes dans un site implique l'utilisation de piège dans des zones privilégiées (ex. garenne pour le lapin) ${ }^{6}$. Par ailleurs, la plupart des gisements livre également d'autres espèces d'herbivores de moyenne taille (ex. bouquetin, renne, cerf), nécessitant alors une pondération de la masse protidique de la part de ces petits gibiers. Ils constituent néanmoins une ressource complémentaire d'importance dans l'alimentation journalier des préhistoriques (Hockett et Bicho 2000 : 721 ; Hockett sous-presse ; Haws et Hockett 2004 ; Aura et al. 2002). Dans ce contexte, le site d'Anecrial se révèle de nouveau particulier, non seulement par la faible fréquence des restes mais aussi par l'absence d'autres mammifères associés. L'aspect écofonctionnel des gisements archéologiques représente alors un critère déterminant pour interpréter le rôle et l'apport réel de petit gibier dans une économie de subsistance (Brugal 2000 ; Cochard et Brugal 2004 ; Hockett et Haws 2003).

II est pertinent de relever la variabilité des échantillons provenant de gisements anthropiques, non seulement en termes d'abondance stricte mais aussi sur les fréquences des parties squelettiques. Une analyse de ces distributions selon cinq grandes classes anatomiques (en \% NME) est proposé pour quelques sites du Paléolithique Supérieur (fig. 8). Globalement, on constate une bonne représentation des os longs des membres avec cependant une plus forte fréquence des éléments postérieurs ; les restes crâniens sont peu nombreux. Les variations les plus importantes concernent le squelette axial et les parties distales des membres (métapodes, phalanges). Ces derniers, de valeur nutritive nulle, sont plus nombreux dans les gisements livrant une grande quantité de restes, qui dénotent des durées d'occupation vraisemblablement plus longues et donc des spectres d'activités sur les carcasses plus larges. Leur moindre fréquence dans des sites moins denses serait en relation avec des traitements différenciés portant sur la fourrure de ces petits animaux. La peau, englobant les bas de pattes, constitue un sous-produit recherché, non abandonné sur les lieux de consommation mais mis de coté et emporté lors du départ des groupes humains. La durée des séjours peut alors déterminer l'usage in situ ou non de cette ressource qui peut être considérée comme un objectif supplémentaire de l'acquisition de ce petit gibier, voire posséder un statut particulier (Fontana 2004).

L'absence du squelette axial semble le cas de figure le plus courant dans les accumulations anthropiques (Hockett et Haws 2002 : tabl.II) et il paraît difficile d'envisager un transport sélectif des carcasses depuis les lieux d'obtention vers le site. Sa fréquence est toutefois relativement forte à Anecrial (c.2) ainsi que dans le gisement magdalénien de Gazel (fig. 8). Ces distributions désignent vraisemblablement une utilisation différentielle des carcasses selon les gisements : soit rejet hors de la zone d'activités, voire un transport hors du site dans le cas d'une consommation différée ; soit, consommation intensive avec écrasement systématique des vertèbres afin de former un broyât encore riche d'un point de vue alimentaire. Ce déficit pourrait également être la conséquence d'un charognage secondaire de la part de petits camivores, spécialement sur les vertèbres qui présentent encore des éléments nutritifs attractifs pour des camivores. L'hypothèse d'une préparation culinaire particulière nous apparaît cependant comme la plus probable, et mériterait d'être confrontée à d'autres obsenations d'ordre ethnographique par exemple?.

Quoi qu'il en soit, cette analyse des représentations squelettiques est liminaire et nécessiterait des groupements plus détaillés entre éléments anatomiques, agrémentée de considérations taphonomiques précises (sur les questions de conservation différentielle jusqu'aux méthodes de fouilles et de tamisage). Elle a pour but de souligner la variabilité dans la structure des assemblages de léporidés produits par l'activité cynégétique humaine. Cette acquisition apparaît directement corrélée à la fonction des gisements ; fonctionnalité appréhendée en terme de taille et composition des groupes humains, du degré de mobilité et de l'intensité des occupations. Ces facteurs déterminent alors l'importance de la prédation sur l'ensemble des ressources localement disponibles.

Les ensembles d'Anecrial restent relativement originaux et dénotent sans conteste de la brièveté des séjours paléolithiques dans cette cavité. L'examen archéozoologique permet de compléter ou pondérer les jeux d'hypothèses formulées par J. Zilhao (1997 : vol.2 , p.127-144) sur la nature du site au moment de l'occupation de la couche 2. L'interprétation retenue par cet auteur définit un seul épisode, durant une nuit, par un groupe de trois individus réalisant des activités de taille autour d'un foyer. La durée et le nombre d'occupations, le nombre d'individus ou de

(6) Outre le filet ou le lacet, une méthode simple consiste à placer un bouchon de branches dans les terriers, à longueur de bras, et à acculer l'animal lors de sa retraite (D.Helmer, comm.pers.).

(7) Cette technique serait documentée dans les sociétés indiennes d'Amérique du Nord (Hocket 1995 et comm.pers.). 


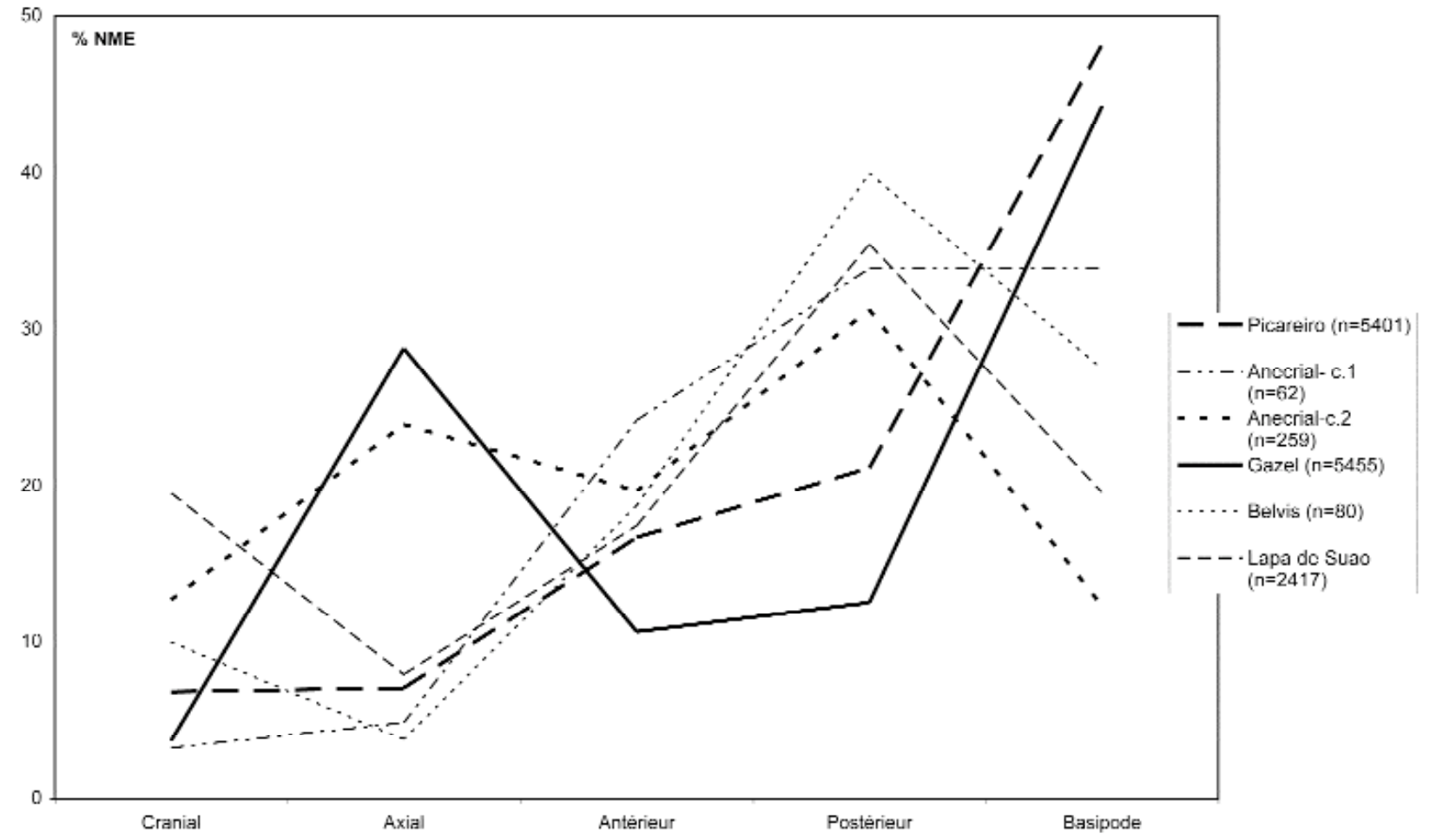

Figure 8 - Comparaison suivant cinq grandes classes anatomiques des représentations squelettiques (\%NME) de lagomorphes de quelques gisements paléolithiques.

Picareiro (niveaux cumulés) : Hockett et Bicho 2000 ; Gazel et Belvis : Fontana 1998 ; Lapa do Suao : Valente 2000. Classes anatomiques = cranial (excluant les fragments crâniens), axial (vertèbres, cotes, excluant le sacrum), os longs antérieurs et postérieurs (incluant calcanéum et talus pour le dernier), basipode (essentiellement métapodes et phalanges).

Figure 8 - Comparison of lagomorph's skeletal parts according to five main categories (\%MNE) for some Paleolithic sites.

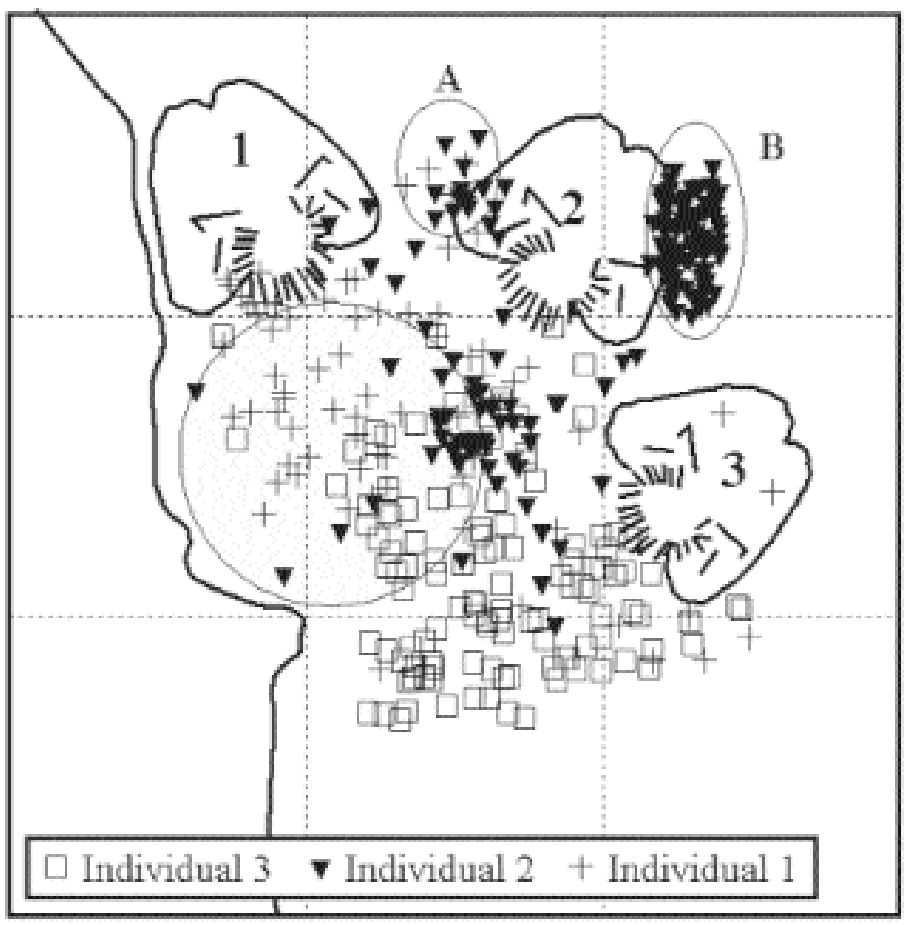

Figure 9 - Interprétation générale de la couche 2 d'Anecrial (Gravettien terminal), suivant la distribution des vestiges lithiques et les remontages effectués (d'après Almeida et al. souspresse : fig. 9).

Cercle = emplacement du foyer; symboles $=$ dispersion de trois types de matières premières lithiques indiquant trois postes de taille (trois individus : 1 à 3 ); $A$ et $B=$ zone de concentration.

Figure 9 - General interpretation of layer 2 of Anecrial (Terminal Gravetian), with spatial distribution of lithic (from Almeida et al. ss-pr. : fig. 9). 
groupes différents, l'emplacement de taille forment le support d'alternatives fonctionnelles. Le gisement est considéré comme un campement transitoire, lieu faisant partie de l'itinéraire d'un groupe de chasseurs voyageant dans un but logistique de chasse ou de reconnaissance, et transportant avec eux une réserve de matière lithique sous forme de blocs de quartz, quartzite et silex ; ces matières premières n'existant pas dans l'environnement proche.

On ne peut que confirmer la plupart de ces observations et nous pensons également que l'occupation désigne un seul et unique moment. Toutefois, l'interprétation des restes de bouquetin comme morceaux (snack) apportés dans un bagage et utilisés dans la confection d'un «bouillon», ne peut être retenue. En effet, ces restes correspondent à des résidus dégradés de séjours antérieurs de groupes humains différents, comme l'a suggéré l'analyse taphonomique, confirmée par la datation obtenue ensuite sur ces ossements. Les lagomorphes, et leur faible nombre, supposent un séjour court, de type bivouac, par un groupe restreint de personnes. De plus, la position du foyer (c.2) contre la paroi et la mise en évidence de trois postes de taille autour de celui-ci, permettent d'avancer un nombre minimal de trois (fig. 9) à maximal de cinq - six personnes (groupe familial ?). Ceux-ci ont consommé au moins 15 lapins dont la prise probable par piégeage assure un séjour relativement court, de l'ordre de quelques nuits. Si l'on considère la prise de quatre lapins dans la couche 1 comme un bivouac d'une nuit (soit 1 jour +1 nuit, 24h), la présence de 15 individus dans la couche 2 impliquerait alors entre trois et quatre 'jour + nuit' (à taille de groupe constant). Cette estimation est aussi confortée par le spectre lithique. Ce calcul simpliste permet en définitive de se figurer une certaine réalité socio-économique. Les deux occupations d'Anecrial sont de même nature, présentant une même finalité de séjours occasionnels s'intégrant dans une mobilité, ou cycle de vie, parfaitement organisée et planifiée.

Le traitement des animaux est différentiel suivant les parties anatomiques: si les membres font l'objet d'une consommation in situ avec destruction des extrémités pour recueillir la moelle, il apparaît que le squelette axial reste peu abondant ainsi que les extrémités des membres. Le rejet des restes vers le foyer semble assez systématique et expliquerait alors les débuts de crémation observée dans un foyer peu vif (couleur marron sur les ossements). La conservation en vue d'une consommation différée des râbles (vertèbres) et le stockage des peaux sont également envisagés. On ne peut toutefois exclure un apport de parties de carcasses de lapins provenant d'une autre zone exploitée lors de déplacements des groupes humains. Ainsi, il est délicat d'élaborer une séquence trop réaliste d'actions précises concernant les moments de collecte du bois, taille des roches, pose des pièges ou dernier repas (Zilhao 1997 : 142).

L'aspect transitoire des séjours à Anecrial et les autres sites connus pour cette même période dans la région, convergent vers un modèle commun. L'exemple d'Anecrial suggère une mobilité de groupes humains de petite taille engagés dans une économie de type logistique déterminée par des facteurs saisonniers. La nature de telles occupations correspond à des environnements climatiques particuliers et, la fonction de ces sites doit alors intégrer l'existence des potentialités en ressources exploitables dans le milieu. Les dates obtenues pour la couche 2 d'Anecrial (Gravettien terminal) sont proches d'une période d'instabilité climatique, contemporaine du dernier maximum glaciaire ; elle est plus précisément corrélée avec un épisode froid enregistré dans les carottes de l'Atlantique Nord et que l'on retrouve également dans les carottes au large des côtes portugaises. II s'agit des événements d'Heinrich (décharge d'iceberg), en particulier le H2 centré autour de 21-22 ka uncal. BP. Ces événements (Thouveny et al. 2000) ont une durée estimée entre 500 à 2000 ans, durée suffisante pour déséquilibrer les zoocœnoses. D'autres gisements archéologiques en cavité, livrant des faunes et proches géographiquement d'Anecrial, se placent dans cet intervalle de temps (Brugal et Valente souspresse) :

- Gravettien et Proto-solutréen de Buraca Escura, c.2, (Pombal): $22700 \pm 240$ et $21820 \pm 200$ ans BP (Aubry et al. 2001) ;

- Solutréen de Caldeirao, c.H (Tomar): $19900 \pm 260$ et $20530 \pm 270$ ans BP (Zilhao 1997 ; Davis 2002);

- une date de $20250 \pm 320$ ans BP pour des niveaux paléolithiques supérieurs (Aurignacien/Gravettien final) de la grotte de Salemas (Loures) reste peu fiable selon le contexte chrono-culturel (Zilhao 1997 et comm.pers.).

Dans tous ces cas, il s'agit d'occupations de très courte durée dans des grottes étroites, de petites dimensions. L'abri de Lagar Velho (Leiria) livre également des installations du Gravettien terminal au Solutréen moyen (TP06 et TP09) datées entre $21180 \pm 240$ et $20220 \pm 180$ ans BP (Zilhao et Trinkaus 2002). Le matériel faunique y est relativement plus dense avec une plus grande diversité spécifique bien que représentant peu d'individus ; les lagomorphes dominent largement ces spectres (MorenoGarcia et Pimenta 2002). De manière générale, ces gisements ne livrent jamais d'ensembles fossiles très abondants et les paléoenvironnements (données anthracologiques) indiquent toujours des paysages ouverts sous un climat relativement frais. Dans une étude des peuplements mammaliens au Portugal (Brugal et Valente sous-presse), la succession des associations animales est précisée pour le dernier glaciaire. La période 22-18 ka montre l'abondance du cerf et du cheval, suivi par le bouquetin et enfin l'aurochs et le sanglier. Chez les camivores, lynx pardelle et renard dominent suivis par le loup, et les grands prédateurs (lion, panthère, hyène) deviennent rares avant leur disparition définitive de ces régions après $18 \mathrm{Ka}$. On peut remarquer que c'est durant cette même période que les groupes préhistoriques développent l'acquisition de petit gibier comme les lagomorphes, alors que les camivores de moyenne taille (lynx, renard), dirigés vers ces mêmes proies, sont également abondants. Cette convergence est intéressante, suggérant un même mécanisme sous-jacent se traduisant par un ajustement faunique, comme par exemple une diminution des ongulés concomitante d'une «explosion» des léporidés ? La fluctuation des gibiers, sous contrôle climatique, entraînera des modèles d'établissement et de gestion des territoires adaptés de la part des préhistoriques. 
L'étude archéozoologique récente de l'un de ces sites (Buraca Escura : Aubry et al. 2001), montre l'exploitation marginale d'herbivores par les hommes (cheval, cerf) alors que la plupart des restes osseux $(79 \%$ du NRD total : Capra) a été apportée et modifiée par le lynx pardelle. Cependant, on note la quasi-absence de lagomorphes dans ce gisement malgré la présence de ce félin connu pour sa spécialisation sur ce type de proie (Delibes 1987). Ce fait souligne l'importance de facteurs locaux dans la disponibilité de catégories de gibier. Globalement, les activités humaines en cavités paraissent toujours discrètes (faible abondance lithique et faunique), avec des fréquentations épisodiques plutôt que des installations concertées, complétées par une exploitation locale des ressources. Cette image différe des occupations de plein-air pour les mêmes périodes considérées comme sites résidentiels, avec des registres lithiques plus abondants mais une faune, malheureusement, non conservée (Zilhao 1997 ; Thacker 1996).

Durant ces périodes, les populations d'ongulés devaient être plus dispersées (sensu diversité et densité) en raison à la fois des refroidissements 'événementiels' du demier glaciaire (type Heinrich ou Dansgaard-Oeschger) et d'un fort cloisonnement géomorphologique - zone côtière plus étendue en rapport avec la baisse du niveau marin (Dias $2004)^{8}$, plaines et vallées, reliefs et réseau hydrographique, etc. La variation des ressources (animales, végétales et lithiques) et leurs acquisitions, exacerbée dans un rythme saisonnier, induit généralement une plus grande mobilité et une utilisation différentielle des régions avec un peuplement plus diffus. Le site d'Anecrial est un bon exemple d'adaptation des groupes aux changements environnementaux s'exprimant en terme de stratégie d'acquisition de petit gibier et de mobilité.

\section{CONCLUSION}

Une double problématique guide cette contribution, concemant la mise en place des exploitations de petit gibier en rapport avec la nature des gisements abordée sous un angle quantitatif (sites 'pauvre' vs 'riche') mais débouchant sur des fonctionnalités socio-économiques. Le contexte ibérique apporte aussi une spécificité, notamment climatique et biocénotique. Le site en grotte d'Anecrial représente deux niveaux contenant de faible quantité de vestiges lithiques et fauniques. Les occupations humaines, attribuées au Gravettien terminal et au Solutréen (Almeida 2000 ; Almeida et al. sous-presse), se trouvent sur des surfaces réduites avec des activités de taille et de consommation réalisées autour de foyers assez importants mais peu aménagés. Les ensembles fauniques sont quasiment mono-spécifiques avec un petit gibier, le lapin, vraisemblablement acquis par la pose de pièges aux alentours du site et/ou capturé lors du déplacement. Les restes associés de Caprinés et quelques artefacts lithiques, correspondent à une fréquentation plus ancienne de la cavité, démontrée par l'analyse taphonomique, la distribution des vestiges et un complément de datation. Cette situation fournit une bonne illustration des mélanges toujours possibles dans des niveaux supposés intègres et homogènes. L'étude archéozoologique permet en outre de préciser un certain nombre de points sur la fonction du gisement grâce aux interprétations sur l'acquisition, la consommation et le traitement différentiel des carcasses de léporidés. Elle apporte de nouvelles données sur la variabilité des comportements humains vis à vis d'une ressource spécifique de petite taille.

Ces résultats permettent de préciser les types d'occupations d'Anecrial. Dans les deux cas, elles correspondraient à un bivouac d'un petit groupe d'individus tournés vers l'exploitation exclusive d'un petit gibier localement abondant (opportunisme?) ; la facilité d'acquisition allant de pair avec la brièveté du séjour et n'impliquant pas pour autant une spécialisation. Le groupe est très mobile et les déplacements sont relativement planifiés (sensu prévision) en raison de l'outillage lithique apporté. L'aspect provisionnel (sensu provision) peut également être souligné avec le transport d'une partie de la nourriture ainsi que de sousproduits animaux (peau). Cette interprétation soulève des questions sur l'exploitation d'ordre logistique de petits mammifères dans une économie paléolithique de subsistance caractérisée par une grande mobilité, créant des lieux provisoires de type station dans des cavités et posant la question de leur relation systémique avec des gisements plus permanents de plein-air ou en abri.

L'abondance de restes de lagomorphes semble liée non seulement à des facteurs naturels comme la diversité et densité des ressources dans un contexte bioclimatique donné mais aussi à la durée des occupations humaines comme reflet de mobilité saisonnière. La fonction des sites, la taille et la structure des groupes humains sont des paramètres pondéraux pour des analyses diachroniques développant des inférences territoriales et démographiques touchant des changements socioculturels majeurs du Paléolithique supérieur. Ils devraient alors être davantage pris en considération avant de proposer des modélisations à grande échelle (Stiner et al. 2000 ; Bietti 2000 ; Brugal 2000).

L'importance des lagomorphes dans le régime alimentaire des groupes humains semble bien démontrée au Portugal, et en Péninsule ibérique, démarrant avec les cultures du Gravettien et du Solutréen et devenant de plus en plus marquée au Magdalénien (par exemple Aura et al. 1998 ; Zilhao 1992, 1997). On peut noter un décalage chronologique avec les régions du sud de la France où cette exploitation ne devient majoritaire qu'à partir du Magdalénien moyen-supérieur (Cochard et Brugal 2004). Cet élargissement alimentaire s'accompagne d'une diversification dans l'exploitation de l'ensemble des ressources (matière premières s.l., sites et paysages) allant de pair avec des transformations techno-symboliques témoignant de nouveaux

(8) Lors du LGM, le niveau marin est à environ -100 à -120 m par rapport à l'actuel, déplaçant la côte en Estrémadure de prés de $35 \mathrm{~km}$ vers l'est (Dias 2004 : fig.2). 
besoins vitaux (sensu démographie) et sociaux (sensu hiérarchisation). L'analyse archéozoologique permet alors, en complément des études typo-technologiques et tracéologiques, considérées dans leurs dimensions spatiales, de mieux interpréter les fonctions socio-économiques des gisements préhistoriques. Le mini-site d'Anecrial représente un cas d'occupation particulièrement bien documenté pouvant constituer une clef de lecture possible pour définir des modèles d'exploitation des territoires et d'org anisation des habitats (settlement pattern). II démontre la nécessité d'intégrer toutes les catégories d'informations, donnant alors toute leur «richesse» aux gisements 'pauvres'.

\section{Remerciements}

Les travaux pluridisciplinaires menés à Lapa do Anecrial ont été rendus possible grâce à un accord de coopération franco-portugais et à des programmes entre le Ministère des Affaires Etrangères et le CNRS, avec I'ICCTI (ex. JNICT), sous la direction de J.Zilhao et du présent auteur. Les analyses et images au MEB ont été prises au «Service Commun de Microscopie Electronique" de l'Université de Provence, Centre de Saint-Charles (Marseille) ; nous remercions vivement R.Notonier et A.Tonello pour leur assistance technique. Merci également aux deux rapporteurs de la revue, P.Fosse et un anonyme, de m'avoir permis de préciser quelques notions.

\section{BIBLIOGRAPHIE}

ALMEIDA F. 2000 - The Terminal Gravettian of Portuguese Estremadura, Ph.D. Dissertation. Southern Methodist University.

ALMEIDA F., BRUGAL J.P., ZILHAO J., PLISSON H. (ss-pr.) An Upper Paleolithic Pompeii : Technology, subistence and paleoethnography at Lapa do Anecrial. (Actas do IV Congresso de Arqueologia Peninsular, Faro, 14-19 Set. 2004), Univers. do Algarve, "From the Mediterranean basin to the Portuguese Atlantic shore: Papers in Honor of A.Marks"

ANDREWS P. 1990 - Owls, Caves and fossils. Chicago : University of Chicago Press.

ANDREWS P., NESBIT EVANS E.M., 1983 - Small mammal bone accumulations produced by mammalian camivores. Paleobiology 9 , 289-307

AUBRY T., BRUGAL J.Ph., CHAUVIERE F.X., FIGUEIRAL I., MOURA M.H., PLISSON H. 2001 - Modalités d'occupations au Paléolithique supérieur dans la grotte de Buraca Escura (Redinha, Pombal, Portugal). Revista Portuguese de Arqueologia, 4(2) : 19-46

AURA J.E., VILLAVERDE V., MORALES M.G., SAINZ C.G., ZILHAO J., STRAUS L.G. 1998 - The PleistoceneHolocene transition in the Iberian peninsula : continuity and change in human adaptations. Quaternary International,49-50: 87-103
AURA J., VILLAVERDE V., PEREZ RIPOLL M., MARTINEZ VALLE R., GUILLEM CALATAYUD P. 2002 - Big game and small prey: paleolithic and epipaleolithic economy from Valencia (Spain). In B.Hockett (ed.), Advances in Paleolithic zooarcheology. Joumal of Archaelogical Method and Theory, 9: 215-268

BEHRENSMEYER, A.K. - 1978 - Taphonomic and ecologic infomation from bone weathering. Paleobiology, 4 (2) : 150-162

BICHO N.F 1994 - The end of the Paleolithic and the Mesolithic in Portugal. Current Anthropology, 35 (5) : 664-674

BICHO N.F., HOCKETT B., HAWS J., BELCHER W. 2000 Hunter-gatherer susbistence at the end of the Pleistocene : preliminary results from Picareiro cave, Central Portugal. Antiquity, $74: 500-506$

BIETTI A. 2000 - comments of Stiner et al., Cument Anthropology, 41(1) : 60-61

BRIDAULT A. 1997 - Broadening and diversification of hunted resources, from the late Palaeolithic to the late Mésolithic, in the north and east of France and the bordering areas. Anthropozoologica, 25-26 : 295-308

BRIDAULT A., CHAIX L. 1995 - Contribution de l'archéozoologie à la caractérisation des modalités d'occupation des sites alpins et jurassiens de l'Epipaléolithique au Néolithique. In L'Eurqpe des derniers Chasseurs, 5é coll.intem UISPP : 547-558

BRUGAL J .P. 2000 - Comments de l'article de M.C.Stiner, N.D.Munro, T.A.Surovell " The tortoise and the hare : smallgame use, the broad spectrum revolution and Palaeolithic demography ", Current Anthropology , 41 (1) : 62-63

BRUGAL J.P., YRAVEDRA SAINZ DE LOS TERREROS 20052006 - Essai sur la biodiversité des associations de grands mammifères à la fin du Pléistocène dans le Sud-Ouest de l'Europe. Munibe, Homenaje a J.Altuna, 57 (1) : 139-162

BRUGAL J.P., VALENTE M.J. (ss-pr.) - Dynamic of large mammalian associations in the pleistocene of Portugal. In "From the Mediterranean basin to the Portuguese Atlantic shore: Papers in Honor of A.Marks, IV Congresso de Arqueologia Peninsular, Univers. Algarve, Faro, 14-19 Sept. 2004,

CALLOU C., 1995 - Modifications de l'aire de répartition du lapin (Oryctolagus cuniculus) en France et en Espagne, du Pléistocène à l'époque actuelle. Etat de la question. Anthropozoologica, 21: 95-113.

CHALINE J. (ss.la dir.) 1974 - Les proies des rapaces, petits mammifères et leur environnement. Paris : Doin éd., 141p.

CHARLES R., JACOBI R.M. 1994 - The lateglacial fauna from the Robin Hood cave, Creswell grags : a re-assessment. Oxford Journal of Archaeology, 13(1) :1-32 
COCHARD D. 1999 - Etude taphonomique des Léporidés du niveau supérieur du diverticule des Rochers de Villeneuve (Jonzac, Indre). In Rapport de Fouille Programmé. C. Beauval (dir.). Poitiers: Service Régional d'Archéologie.

COCHARD D. 2000 - Etude taphonomique des Lagomorphes de Coudoulous II (Lot, France). In Rapport de Fouille Programmée, J.P. Brugal (dir.), Toulouse : Service Régional d'Archéologie

COCHARD D. 2004 - Les léporidés dans la subsistance paléolithique du Sud de la France. Thèse de Doctorat, Université de Bordeaux I, 356p.

COCHARD D., J.P.BRUGAL 2004 - Importance des fonctions de sites dans les accumulations paléolithiques de Léporidés. In Petits Animaux et Sociétés Humaines - Du complément alimentaire aux ressources utilitaires, J.P.B rugal \& J.Desse (ss la dir.), Actes des XXIVe Rencontres Internationales d'Archéologie et d'Histoire d'Antibes, éd. APDCA : 283-296

CRUZ-URIBE K., R.KLEIN 1998 - Hyrax and Hare bones from modern south african eagle roosts and the detection of eagle involvement in fossil bone assemblages. Journal of Archaeological Science, 25 : 135-147.

DAVIS S.J.M. 2002 - The mammals and birds from the Gruta do Caldeirão, Portugal. Revista Portuguesa de Arqueologia, 5 (2), p. 29 - 98.

DELIBES M. 1987 (réimpr.) - El lince iberico. Donana, Acta Vertebrata, 7 (3) : 128p.

DELPECH F., RIGAUD J.Ph. 1974 - Etude de la fragmentation et de la répartition des restes osseux dans un niveau d'habitat paléolithique. In Premier Coll.Intern. sur I'Industrie de l'os dans la Préhistoire, H.Camps-Fabrer (org.), éd. Université de Provence : 47-55

DIAS ALVEIRINHO J.M. 2004 - A historia da evoluçao do litoral português nos ultimos vinte milenios. Evoluçao Geohistorica do Litoral Português e Fenomenos correlativos : Geologia, Historia, Arqueologia e Climatologia, Lisboa : 157-170

FIGUEIRAL I.1995 - Charcoal analysis and the history of Pinus pinaster (cluster pine) in Portugal. Review of Palaeobotany and Palynology , 89 : 441-454

FIGUEIRAL I., TERRAL J.-F. 2002 - Late Quaternary refugia of Mediterranean taxa in the Portuguese Estremadura : charcoal based palaeovegetation and climatic reconstruction. Quaternary Science Reviews , 21 : 549-558

FONTANA L. 1998 - Mobilité et subsistance au Magdalénien dans le Languedoc occidental et le Roussillon. Univers. Paris I, Panthéon-Sorbonne : Doct. Préhistoire, 2 vol. (polycopié)
FONTANA L. 1999 - Mobilité et subsistance au Magdalénien dans le Bassin de l'Aude. Bulletin de la S.P.F. $96,175-190$.

FONTANA L. 2004 - Le statut du lièvre variable (Lepus timidus) en Europe occidentale au Magdalénien : premiers bilans et perspectives. In Petits Animaux et Sociétés Humaines - Du complément alimentaire aux ressources utilitaires, J.P.B nugal \& J.Desse (ss la dir.), Actes des XXIV ${ }^{\circ}$ Rencontres Internationales d'Archéologie et d'Histoire d'Antibes, éd. APDCA : 297-312

GRAYSON D.K., DELPECH F. 1998 - Changing diet breadth in the early upper paleolithic of southwestern France. Journal of Archaeological Science , 25 : 1119-1129

HAWS J.A., HOCKETT B.S. 2004 - Theoretical perspectives on the dietary role of small animals in human evolution. In Petits Animaux et Sociétés Humaines - Du complément alimentaire aux ressources utilitaires, J.P.Brugal \& J.Desse (ss la dir.), Actes des XXIVe Rencontres Internationales d'Archéologie et d'Histoire d'Antibes, ed.APDCA : 533-544

HOCKETT B.S. 1989 - Archaeological significance of rabbit-raptor interactions in Southern California. North American archaeologist $10,123-139$.

HOCKETT B.S. 1991 - Toward distinguishing human and raptor patterning on leporid bones. American Antiquity 56, 667-679.

HOCKETT B.S. 1994 - A descriptive reanalysis of the leporid bones from Hogup cave, Utah. Joumal of California and Great Basin Anthropology, 16(1) : 106-117

HOCKETT B.S. 1995 - Comparison of leporid bones in raptor pellets, raptor nests, and archaeological sites in the great basin. North american archaeologist 16 , 223-238.

HOCKETT B.S. 1996 - Corroded, thinned and polished bones created by golden eagles (Aquila chrysaetos) : taphonomic implications for archaeological interpretations. Journal of Archaeological Science $23,587-591$.

HOCKETT B.S. 1999 - Taphonomy of a carnivore-accumulated rabbit bone assemblages from Picareiro cave, Central Portugal. Journal of Iberian Archaeology, 1 : 225-230

HOCKETT B.S. (ss-pr.) - Small faunal use during the middle and late pleistocene of Portugal: a nutritional ecology perspective. Proceeding of the $4^{\text {th }}$ Iberian Archaeology Congress, Faro, Portugal

HOCKETT B.S., BICHO N.F. 2000 - The rabbits of Picareiro cave : small mammal hunting during the late upper Palaeolithic in the Portuguese Estremadura. Journal of Archaeological Science, $27: 715-723$

HOCKETT B., HAWS J.A. 2002 - Taphonomic and methodological perspectives of leporid hunting during the Upper 
Paleolithic of the WestemMediterranean basin. Joumal of Archaeological Method and Theory, 9(3): 269-302.

HOCKETT B.S., HAWS J. 2003 - Nutritional ecology and diachronic trends in Paleolithic diet and health. Evolutionary Anthropology, 12: 211-216

ISAAC G., HARRIS J.W.K., MARSHALL F. 1981 - Small is informative : the application of the study of mini-sites and least effort criteria in the interpretation of the early pleistocene archaeological record at Koobi Fora, Kenya. Union Internacional de Ciencias Prehsitoricas y Protohistoricas, Actas del X Congreso (UNESCO, Mexico), pp.101-119

LUPO K.D., SCHMITT D.N. 2002 - Upper palaeolithic nethunting, small prey exploitation and women's work effort : a view from the ethnographic and ethnoarchaeological record of the Congo basin. Journal of Archaeological Method and theory, 9(2) : 147-179

MOREL P., 1986 - Quelques polis naturels d'apparence trompeuse. Artefacts,3 : 43-45 (éd. du C.E.D.A.R.)

MORENO-GARCIA M., PIMENTA C.M. 2002 - The paleofaunal context. In J.Zilhao \& E.Trinkaus (éds.), Trabalhos de Arqueologia, Lisboa, 112-131

PEREZ RIPOLL M. 1993 - Las marcas tafonomicos en huesos de lagoformos. Estudio sobre Cuaternario : 227-231

PEREZ RIPOLL M. 2004 -La comsommation humaine des lapins pendant le Paléolithique dans la région de Valencia (Espagne) et l'étude des niveaux gravettiens de La Cova de les Cendres (Alicante). In Petits Animaux et Sociétés Humaines - Du complément alimentaire aux ressources utilitaires, J.P.Brugal \& J.Desse (ss la dir.), Actes des XXIVe Rencontres Internationales d'Archéologie et d'Histoire d'Antibes, éd. APDCA :191-206

QUEIROZ P., LEEUWAARDEN W., MATEUS J. 2002 The Paleovegetational Context in Zilhão J. \& Trinkaus E. (eds.) Portrait of the artist as a child. The Gravettian Human Skeleton from the Abrigo do Lagar Velho and its Archaeological Context. Trabalhos de Arqueologia 22. Lisbonne, Instituto Português de Arqueologia : 92-111

QUIRT-BOOTH T., CRUZ-URIBE K. 1997 - Analysis of leporid remains from prehistoric Sinuaga sites. Northem Arizona. Journal of Archaeological Science, 24 : 945-960

ROWLEY-CONWY P. 1992 - The early neolithic animal bones from Gruta do Caldeirao. In Gruta do Caldeirao. $O$ Neolitico Antigo, J.Zilhao (éd.), Lisboa, Instituto Portugues do Patrimonio Arquitectonico e Arqueologico : 231-257

SANCHIS SERRA A. 2000 - Los restos de Oryctolagus cuniculus en las tafocenosis de Bubo bubo y Vulpes vulpes y su aplicacion a la caracterizacion del registro faunistico arqueologico. Sangvntvm,32 : 31-49
SERONIE-VIVIEN M.-R. 1994 - Données sur le lapin azilien. Le matériel du gisement de Pégourié, Caniac-duCausse (Lot). Bulletin de la Société Préhistorique Française $91,378-384$

SCHMITT D.N., JUELL K.E. 1994 - Toward the identification of Coyote scatological faunal accumulations in a rchaeological contexts. Joumal of Archaeological Science, $12: 249-262$.

SHAFFER B.S. 1992 - Interpretation of Gopher remains from southwestern archaeological assemblages. American Antiquity, 57(4) : 683-691

STINER M.C. 1994 - Honor among thieves. A zooarchaeological study of neandertal ecology. Princeton, New Jersey: Princeton University Press.

STINER M.C. 2004 - Small game use and expanding diet breath in the eastern mediterranean basin during the Palaeolithic. In Petits Animaux et Sociétés Humaines - Du complément alimentaire aux ressources utilitaires, J.P.B rugal \& J.Desse (ss la dir.), Actes des XXIVe Rencontres Internationales d'Archéologie et d'Histoire d'Antibes, éd. APDCA : 499-513

STINER M.C., MUNRO N.D., SUROVELL T.A., TCHERNOV E., BAR-YOSEF O. 1999 - Paleolithic population growth pulses evidenced by small animal exploitation. Science, $283: 190-194$.

STINER M.C., MUNRO N.D., SUROVELL T.A. 2000 - Smallgame use, the broad spectrum revoution, and the paleolithic demography. Current Anthropology, 41(1) : 39-73

STRAUS L.G. 1991 - Southwestern Europe at the last glacial maximum. Current Anthropology, 32 : 189-199

THACKER P. 1996 - Hunter-gatherer lithic economy and settlement systems: Understanding regional assemblage variability in the Upper Paleolithic of Portuguese Estremadura. In Odell, G. (ed.), Stone Tools: Theoretical Insights into Human Prehistory, Plenum Press, New York, p. 110-124

THOUVENY N., MORENO E., DELANGHE D., CANDON L., LANCELOT Y., SHACKLETON N.J. 2000 - Rock magnetic detection of distal ice-rafted debries : clue for the identification of Heinrich layers on the Portuguese margin. Earth and Planetary Science Letters, $180: 61-75$

VALENTE M.J. 2000 - Arqueozoologia e tafonomia em contexto paleolitico. A gruta do Pego do Diabo (Loures). Diss. Mestrado, Univers. Lisboa : 129p.+pl.

VIGNE J.-D., MARINVAL-VIGNE M.-C., 1983 - Méthode pour la mise en évidence de la consommation du petit gibier. In Animals and archaeology : 1 - Hunters and their prey, J.Clutton-Brock, C. Grigson (eds), British Archeological Reports, intem.ser.163 : 239-242 
YELLEN J.E.1991 - Small mammals: !Kung San utilization and the production of faunal assemblages. Journal of Anthropological Archaeology ,10(1): 1-26.

ZILHAO J. 1991 - Le solutréen du Portugal : environnement, chronologie, industries, peuplement, origines. In Les industries à pointes foliacées du Paléolithique supérieur européen, Kracow 1989, E.R.A.U.L. : 485-501

ZILHAO J. 1992 - Estrategias de povoamento e subsistência no Paleolitico e no Mesolitico de Portugal. In Elefantes, Cienos y Ovicaprinos : economia y aprovechamiento del medio en la Prehistoria de España y Portugal, A.Moure Romanillo (éd.), Santander : Univers. de Cantabria : 149- 162

ZILHAO J. 1997 - O Paleolitico superior da Estremadura portuguesa. Lisboa : Estudos monograficos, ed.Colibri, 2 vol.
ZILHÃO J. , ALMEIDA F. 1996 - Interpretação Tecnológica e Paletnográfica da Ocupação Proto-Solutrense da Lapa do Anecrial (Porto de Mós), Ophiussa,

ZILHÃO J., ALMEIDA F. - 2002 The Archaeological Framework in Zilhão J.; Trinkaus E. (eds.) Portrait of the artist as a child. The Gravettian Human Skeleton from the Abrigo do Lagar Velho and its Archaeological Context. Trabalhos de Arqueologia 22. Lisbonne, Instituto Português de Arqueologia: 29-57

ZILHAO J., TRINKAUS E. (eds.) 2002 - Portrait of the artist as a child - The gravettian human skeleton from the abrigo do Lagar Velho and its archaeological context. Trabalhos de Arqueologia, Lisboa, Instituto Português de Arqueologia, $22: 604 \mathrm{p}$. 
